\title{
Enumeration of Stereoisomers of Chiral and Achiral Derivatives of Monocyclic Cycloalkanes Having Heteromorphous Alkyl Substituents with Distinct Length $k$
}

\author{
Alphonse Emadak ${ }^{*}$, Ibrahim Mbouombouo Ndassa'2, Beauregard Thomas Makon1, \\ Issofa Patouossa ${ }^{1}$, Robert Martin Nemba ${ }^{1}$ \\ ${ }^{1}$ Laboratory of Applied Physical and Analytical Chemistry, Department of Inorganic Chemistry, Faculty of Sciences, \\ University of Yaounde 1, Yaounde, Cameroon \\ ${ }^{2}$ Laboratory of Chemistry, Department of Chemistry, High Teacher Training College, University of Yaounde 1, \\ Yaounde, Cameroon \\ Email: *emadak@yahoo.com
}

How to cite this paper: Emadak, A., Ndassa, I.M., Makon, B.T., Patouossa, I. and Nemba, R.M. (2019) Enumeration of Stereoisomers of Chiral and Achiral Derivatives of Monocyclic Cycloalkanes Having Heteromorphous Alkyl Substituents with Distinct Length $k$. Computational Chemistry, 7, 72-93. https://doi.org/10.4236/cc.2019.73006

Received: April 20, 2019

Accepted: July 28, 2019

Published: July 31, 2019

Copyright $\odot 2019$ by author(s) and Scientific Research Publishing Inc. This work is licensed under the Creative Commons Attribution International License (CC BY 4.0).

http://creativecommons.org/licenses/by/4.0/

c) (†) Open Access

\section{Abstract}

A combinatorial method based on the determination of the averaged weight of permutations controlling the chirality/achirality fittingness of $2 n$ substitution sites of the monocyclic cycloalkane allows to obtain generalized functional equations for direct enumeration of enantiomers pairs and achiral skeletons of any derivatives of monocyclic cycloalkanes having heteromorphic alkyl substituents with the distinct length $k$ with the empirical formula $C_{n} H_{m_{0}}\left(R_{k_{1}}\right)_{m_{1}} \cdots\left(R_{k_{\alpha}}\right)_{m_{\alpha}} \cdots\left(R_{k_{\zeta}}\right)_{m_{\zeta}}$, wherein at least two alkyl groups $R_{k_{\alpha}}=C_{k_{\alpha}} H_{2 k_{\alpha}+1}$ of the distinct size $k_{\alpha(1 \leq \alpha \leq \zeta)}$ each. $m_{\alpha}$ is the number of alkyl radicals $R_{k_{\alpha}}$ of the system $C_{n} H_{m_{0}}\left(R_{k_{1}}\right)_{m_{1}} \cdots\left(R_{k_{\alpha}}\right)_{m_{\alpha}} \cdots\left(R_{k_{\zeta}}\right)_{m_{\zeta}}$ verifying the relation $\sum_{\alpha=1}^{\zeta} m_{\alpha}+m_{0}=2 n$. The integer sequences of enantiomer pairs and achiral skeletons are given for substituted derivatives of monocyclic cycloalkane for $n=3,4$ and $k=3,4,5$. The composite stereoisomerism of this particular compound is also highlighted.

\section{Keywords}

Enumeration, Stereoisomer, Chirality, Achirality, Monocyclic Cycloalkane, Order of Alkyl Trees, Polyalkylation 


\section{Introduction}

From the 1800s to 1870 , the enumeration of isomers in chemistry consisted of a manual enumeration based on the so-called "draw and count" method which consists of drawing and counting all the possible molecular structures of a given organic compound in two dimensions or a plan. This exhausting exercise used to expose certain risks of repetition and/or omission of structures, especially for large molecules [1].

Chemists sorted out these difficulties of enumeration of chemical structures by developing methods and models of enumeration using not only mathematical theories (group theory, graph theory, etc.) but also more and more information technology tools (computers, softwares, etc.). This allowed to first generate several methods of enumeration of the isomers of constitution or function [2] [3] [4] [5] [6] and in a second step of the enumeration of isomers of substitution then the elaboration of the direct formulas of quantitative determination stereoisomers [7]-[16]. Concretely, the computation of the number of stereoisomers of the homopolysubstituted and heteropolysubstituted monocyclic cycloalkane has been the subject of several studies [17]-[22]. Nemba et al. have dealt in this dynamic with the problem of enumeration of substituted monocycloalkanes with homomorphic alkyl substituents [23] and Emadak et al. have enumerated the enumeration of substituted monocyclic cycloalkanes having sterically heteromorphic alkyl substituents of identical $k$ order [24].

In this work, we are interested in determining the number of enantiomeric pairs and achiral skeletons of cycloalkanes with heteromorphic alkyl substituents of distinct order $k$. To simplify the language, we will speak of heteropolyalkylation of monocyclic cycloalkane with alkyl radicals of distinct $k$ size. Beyond the direct formulas of computation established, this work highlights, on the one hand, a composite stereoisomerism due to the intrinsic chirality of the alkyl trees and to the extrinsic chirality induced by their positioning or placement on the monocycloalkane, on the other hand, the total number of different stereoisomers increases correlatively with the number of chiral centers present in the molecular graph and finally the concepts of pseudochirality, pseudosymmetry and enantiomeric mirror in the molecules of this family of saturated hydrocarbon compounds is also elucidated.

\section{Definitions and Mathematical Formulation}

\subsection{Stereograph Composition of $G_{k}^{\gamma}$}

Let us consider the system $C_{n} H_{m_{0}}\left(R_{k_{1}}\right)_{m_{1}} \cdots\left(R_{k_{\alpha}}\right)_{m_{\alpha}} \cdots\left(R_{k_{\zeta}}\right)_{m_{\zeta}}$, the empirical formula of the heteropolyalkylated monocyclic cycloalkane having heteromorphous alkyl substituents including at least two alkyl groups noted $R_{k_{\alpha}}=C_{k_{\alpha}} H_{2 k_{\alpha}+1}$, having the distinct order, size or length $k_{\alpha}$, with $(1 \leq \alpha \leq \zeta) . m_{\alpha}$ is the number of alkyl radicals $R_{k_{\alpha}}$ of the system $C_{n} H_{m_{0}}\left(R_{k_{1}}\right)_{m_{1}} \cdots\left(R_{k_{\alpha}}\right)_{m_{\alpha}} \cdots\left(R_{k_{\zeta}}\right)_{m_{\zeta}}$ where 
$\sum_{\alpha=1}^{\zeta} m_{\alpha}+m_{0}=2 n$.

The molecular composite stereograph $G_{k_{\alpha}}$ of the system

$$
C_{n} H_{m_{0}}\left(R_{k_{1}}\right)_{m_{1}} \cdots\left(R_{k_{\alpha}}\right)_{m_{\alpha}} \cdots\left(R_{k_{\zeta}}\right)_{m_{\zeta}}
$$

is obtained by connecting the roots of the graphs of the distinct planted steric trees $R_{k_{\alpha}}$ to the sites or positions of substitution of the stereograph $G$ of the parent molecule of the monocyclic cycloalkane $C_{n} H_{2 n}$ described in our previous work [24]. The mathematical and graphical interpretations of this composition of the graph $G$ and the graphs $R_{k_{\alpha}}$ to obtain $G_{k_{\alpha}}$ are given respectively by the relation (1) and Figure 1 .

$$
G_{k_{\alpha}}=G \circ R_{k_{\alpha}(1 \leq \alpha \leq \zeta)}=C_{n} H_{m_{0}}\left(R_{k_{1}}\right)_{m_{1}} \cdots\left(R_{k_{\alpha}}\right)_{m_{\alpha}} \cdots\left(R_{k_{\zeta}}\right)_{m_{\zeta}}
$$

$G_{k_{\alpha}}$ is the stereograph of a monocyclic cycloalkane heteropolyalkylated having alkyl radicals $R_{k_{\alpha}}$ of distinct size $k$. The approach used to enumerate stereoisomers of the system $C_{n} H_{m_{0}}\left(R_{k_{1}}\right)_{m_{1}} \cdots\left(R_{k_{\alpha}}\right)_{m_{\alpha}} \cdots\left(R_{k_{\zeta}}\right)_{m_{\zeta}}$ is to first determine the number of heteromorphic combinations of distinct alkyl radicals of orders $k_{\alpha}$, with $(1 \leq \alpha \leq \zeta)$, and then to carry out the product of the latter by the results of the enumeration of the stereoisomers of the homopolysubstituted and/or heteropolysubstituted monocyclic cycloalkane obtained in our previous work [21] [22] [25].

\subsection{Calculation of the Number of Combinations of Heteromorphous Alkyl Radicals of Distinct Size $\boldsymbol{k}$}

According to graph theory, any alkyl radical $C_{k} H_{2 k+1}$ is equivalent to a planted steric tree which may have one or more isomers or stereoisomers including the chiral and achiral forms. $s_{k}$ and $p_{k}$ respectively give the total number and the number of steric trees planted of order $k$. We recall that the generating functions which give these numbers were established by Robinson et al. [26] in polynomials' series: $s(x)=\sum_{k} s_{k} x^{k}$ and $p(x)=\sum_{k} p_{k} x^{k} \quad$ for $0 \leq k \leq 14$. Values of $s_{k}, p_{k}$ and $s_{k}-p_{k}$ for $0 \leq k \leq 18$ are computed and compiled in Table 1 .

Graphs of planted steric trees $\left(C_{k} H_{2 k+1}\right)$ can be combined in three sets whose

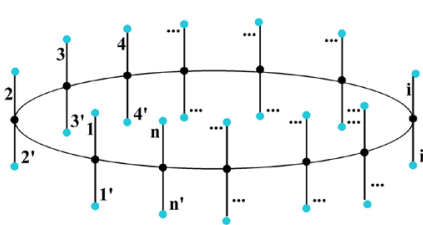

G

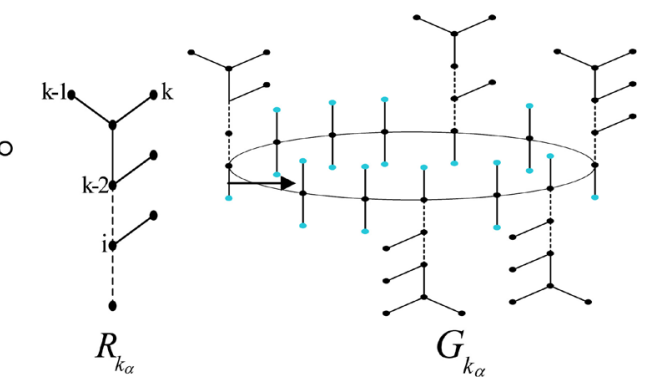

Figure 1. Composition of graph $G$ with distinct planted heteromorphous trees. 
Table 1. Value of $s_{k}, p_{k}$, and $s_{k}-p_{k}$ for $k \leq 18$.

\begin{tabular}{|c|c|c|c|}
\hline$k$ & $s_{k}$ & $p_{k}$ & $s_{k}-p_{k}$ \\
\hline 0 & 1 & 1 & 0 \\
\hline 1 & 1 & 1 & 0 \\
\hline 2 & 1 & 1 & 0 \\
\hline 3 & 2 & 2 & 0 \\
\hline 4 & 5 & 3 & 2 \\
\hline 5 & 11 & 5 & 6 \\
\hline 6 & 28 & 8 & 20 \\
\hline 7 & 74 & 14 & 60 \\
\hline 8 & 199 & 23 & 176 \\
\hline 9 & 551 & 41 & 510 \\
\hline 10 & 1533 & 69 & 1464 \\
\hline 11 & 4436 & 122 & 4314 \\
\hline 12 & 12,832 & 208 & 12,624 \\
\hline 13 & 37,496 & 370 & 37,126 \\
\hline 14 & 110,500 & 636 & 109,864 \\
\hline 15 & 327,420 & 1134 & 326,286 \\
\hline 16 & 979,819 & 1963 & 977,856 \\
\hline 17 & $2,944,873$ & 3505 & $2,941,368$ \\
\hline 18 & $8,896,515$ & 6099 & $8,890,416$ \\
\hline
\end{tabular}

elements are equivalent to stereoisomers of sterically distinct alkyl radicals, namely:

- $E_{a+c}$ is the set of chiral and achiral trees of cardinality $s_{k}$. Elements of $E_{a+c}$ are pairs of enantiomers and achiral skeletons.

- $E_{a}$ is the set of achiral trees of cardinality $p_{k}$ Elements of $E_{a}$ are achiral skeletons.

- $E_{c}$ is the set pairs enantiomers of cardinality $s_{k}-p_{k}$

From these definitions, we can write the following relations:

$$
\left|E_{c+a}\right|=s_{k},\left|E_{a}\right|=p_{k} \Rightarrow\left\{\begin{array}{l}
E_{c+a}=E_{c} \cup E_{a} \\
E_{c} \cap E_{a}=\phi
\end{array}\right.
$$

Considering (2), we have:

$$
\left|E_{c+a}\right|=\left|E_{c}\right|+\left|E_{a}\right| \Rightarrow\left|E_{c}\right|=\left|E_{c+a}\right|-\left|E_{a}\right|
$$

$$
\text { And consequently }\left|E_{c}\right|=s_{k}-p_{k}
$$

Table 2 gives us the graphic representation of some distinct sterical trees of size $k$ identified by letters, $1 \leq k \leq 5$.

Note that a pair of enantiomers is represented by the same letter whose two stereoisomers are differentiated by the apostrophe. 
Table 2. Graphical representation of some planted trees identified by letters of the alphabet.

\begin{tabular}{cc}
\hline Size $\mathbf{k}$ & Alkyl radicals or \\
\hline &
\end{tabular}

3

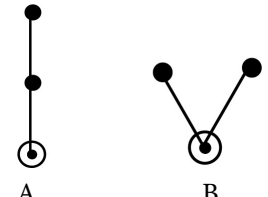

4

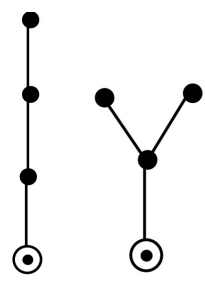

A

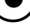

A

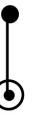

A

B

B

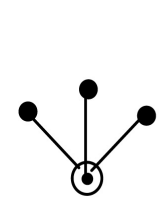

C

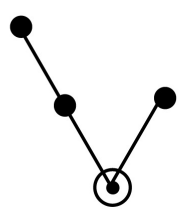

$\mathrm{D}$

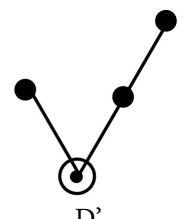

D'

5
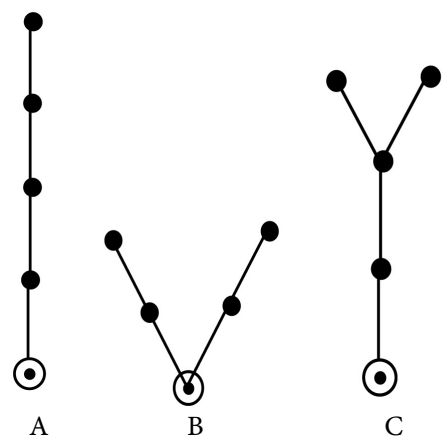

C
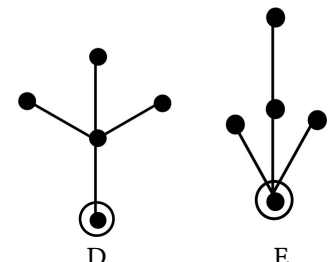

D

E
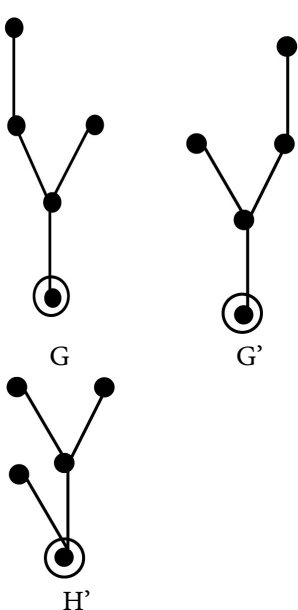

Before calculating the numbers of heteromorphic combinations of distinct alkyl radicals of different order $k$, we first define the different sets of alkyl radicals 
of distinct orders $k_{\alpha}$, with $(1 \leq \alpha \leq \zeta) . I R_{k_{\alpha}}$ is a set constituted of chiral and achiral alkyl radicals having $k_{\alpha}$ carbons. Considering Table 2 illustrating the alkyl radicals of order 1 to 5 below, and assigning $k_{\alpha}$ as an index under each letter corresponding to an alkyl radical having $k_{\alpha}$ carbons, one can set up the following sets:

$$
\begin{gathered}
I R_{k_{1}}=I R_{1}=\left\{A_{1}\right\} \\
I R_{k_{2}}=I R_{2}=\left\{A_{2}\right\} \\
I R_{k_{3}}=I R_{3}=\left\{A_{3}, B_{3}\right\} \\
I R_{k_{4}}=I R_{4}=\left\{A_{4}, B_{4}, C_{4}, D_{4}, D_{4}^{\prime}\right\} \\
I R_{k_{5}}=I R_{5}=\left\{A_{5}, B_{5}, C_{5}, D_{5}, E_{5}, E_{5}^{\prime}, F, F_{5}^{\prime}, G, G_{5}^{\prime}\right\}
\end{gathered}
$$

We can generalize all these sets as follows:

$$
I R_{k_{\alpha}}=\left\{A_{\alpha}, B_{\alpha}, C_{\alpha}, D_{\alpha}, D_{\alpha}^{\prime}, \cdots, Z_{\alpha}, Z_{\alpha}^{\prime}\right\} .
$$

- $I R_{k_{\alpha}}^{a}$ designates a set formed of alkyl radicals only achiral and having the same order $k_{\alpha}$.

Example: $I R_{1}^{a}=\left\{A_{1}\right\}, \quad I R_{2}^{a}=\left\{A_{2}\right\}, \quad I R_{3}^{a}=\left\{A_{3}, B_{3}\right\}, \quad I R_{4}^{a}=\left\{A_{4}, B_{4}, C_{4}\right\}$, $I R_{5}^{a}=\left\{A_{5}, B_{5}, C_{5}, D_{5}, E_{5}\right\}, \cdots$

- $I R_{k_{\alpha}}^{c}$ means a group consisting of only chiral alkyl radicals and having the same order $k_{\alpha}$.

Example: $I R_{4}^{c}=\left\{D_{5}, D_{5}^{\prime}\right\}, I R_{5}^{c}=\left\{F_{5}, F_{5}^{\prime}, G_{5}, G_{5}^{\prime}, H_{5}, H_{5}^{\prime}\right\}, \cdots$

- $\operatorname{CardI} I R_{k_{\alpha}}=s_{k_{\alpha}}$, Card IR $R_{k_{\alpha}}^{a}=p_{k_{\alpha}}$ and $\operatorname{Card} I R_{k_{\alpha}}^{c}=s_{k_{\alpha}}-p_{k_{\alpha}}$.

- A group of substituents or alkyl radicals refers in this chapter to a combination of at least two different substituent types selected from at least two sets of alkyl radicals of order $k_{\alpha}$ different and that composes a substitution.

Example: Heteromorphous substituent groups $A_{1} A_{3}$ and $A_{1} B_{3}$ were formed by combining a radical of $I R_{1}^{a}$ and one of $I R_{3}^{a}$.

- $i$ is the number of substituent types or alkyl radicals that make up a group of substituents or else i-uples. $i_{\alpha}$ is the number of substituent or alkyl radical of order $k_{\alpha}$ chosen in $I R_{k_{\alpha}}, I R_{k_{\alpha}}^{a}$ or $I R_{k_{\alpha}}^{c}$ such that $i_{\alpha} \leq s_{k_{\alpha}}, p_{k_{\alpha}}$ or $s_{k_{\alpha}}-p_{k_{\alpha}}$ and $\sum_{\alpha} i_{\alpha}=i$.

Example: In the group of heteromorphic substituents $A_{1} A_{3} B_{3}, i_{3}=2, i_{1}=1$ and $i=3$; in heteromorphous substituent groups $A_{1} A_{3}$ and $A_{1} B_{3}$ we have $i_{3}=1, i_{1}=1$ and $i=2$.

Following these definitions, we can roll out the algorithm of our method of counting the stereoisomers of the molecular system

$$
C_{n} H_{m_{0}}\left(R_{k_{1}}\right)_{m_{1}} \cdots\left(R_{k_{\alpha}}\right)_{m_{\alpha}} \cdots\left(R_{k_{\zeta}}\right)_{m_{\zeta}}
$$

as follows by first establishing the formula for calculating the number of substituting group or number of $i$-uples of letters identifying the alkyl radicals.

The number of $i$-uples of distinct letters without repetition or groups of subs- 
tituents taken in $I R_{k_{\alpha}}, I R_{k_{\alpha}}^{a}$ or $I R_{k_{\alpha}}^{c}(1 \leq \alpha \leq \zeta)$ in such a way that we have $i$ types of heteromorphic substituents of different orders $k_{\alpha}$ and composing a substitution is given respectively by $N_{k_{1}, k_{2}, \cdots, k_{\alpha}}=\prod_{\alpha}\left(\begin{array}{c}s_{k_{\alpha}} \\ i_{\alpha}\end{array}\right), \quad N_{k_{1}, k_{2}, \cdots, k_{\alpha}}^{a}=\prod_{\alpha}\left(\begin{array}{c}p_{k_{\alpha}} \\ i_{\alpha}\end{array}\right)$ and $N_{k_{1}, k_{2}, \cdots, k_{\alpha}}^{c}=\prod_{\alpha}\left(\begin{array}{c}s_{k_{\alpha}}-p_{k_{\alpha}} \\ i_{\alpha}\end{array}\right)$.

The number of $i$-tuples mixed letter representing heteromorphic combinations of chiral and achiral radicals of distinct orders is given by

$$
N_{k_{1}, k_{2}, \cdots, k_{\alpha}}^{a+c}=N_{k_{1}, k_{2}, \cdots, k_{\alpha}}-N_{k_{1}, k_{2}, \cdots, k_{\alpha}}^{a}-N_{k_{1}, k_{2}, \cdots, k_{\alpha}}^{c} .
$$

\section{Example:}

1) Let us determine the number of substituent groups having 2 types of substituents or even 2-ones of letters of which one is taken in $I R_{3}$ and in $I R_{4}$.

We have $I R_{3}=\left\{A_{3}, B_{3}\right\}, \quad I R_{4}=\left\{A_{4}, B_{4}, C_{4}, D_{4}, D_{4}^{\prime}\right\}, s_{4}=5, p_{4}=3$, $s_{4}-p_{4}=2, i_{4}=1, s_{3}=2, p_{3}=2, s_{3}-p_{3}=0, i_{3}=1$ then

$$
N_{3,4}=\left(\begin{array}{l}
2 \\
1
\end{array}\right) \cdot\left(\begin{array}{l}
5 \\
1
\end{array}\right)=10
$$

which corresponds to the groups of substituents $A_{3} A_{4}, A_{3} B_{4}, A_{3} C_{4}, A_{3} D_{4}, A_{3} D_{4}^{\prime}$, $B_{3} A_{4}, B_{3} B_{4}, B_{3} C_{4}, B_{3} D_{4}, B_{3} D_{4}^{\prime}, N_{3,4}^{c}=0, N_{3,4}^{a}=\left(\begin{array}{l}2 \\ 1\end{array}\right) \cdot\left(\begin{array}{l}3 \\ 1\end{array}\right)=6$ which corresponds to the groups of substituents $A_{3} A_{4}, A_{3} B_{4}, A_{3} C_{4}, B_{3} A_{4}, B_{3} B_{4}, B_{3} C_{4} . \quad N_{3,4}^{a+c}=10-6=4$ which corresponds to the group of substituents $A_{3} D_{4}, A_{3} D_{4}^{\prime}, B_{3} D_{4}, B_{3} D_{4}^{\prime}$.

2) Let us determine the number of substituent groups having 4 substituent types or even 4-tuples of letters of which one is chosen from $I R_{1}$, one in $I R_{2}$ and 2 in $I R_{3}$.

We have $I R_{1}=\left\{A_{1}\right\}, \quad I R_{2}=\left\{A_{2}\right\}$ and $I R_{3}=\left\{A_{3}, B_{3}\right\} ; s_{1}=1, \quad p_{1}=1$, $s_{1}-p_{1}=0, \quad i_{1}=1, \quad s_{2}=1, \quad p_{2}=1, s_{2}-p_{2}=0, i_{2}=1, s_{3}=2, \quad p_{3}=2$, $s_{3}-p_{3}=0, \quad i_{3}=2 ; \quad N_{1,2,3}=N_{1,2,3}^{a}=\left(\begin{array}{l}1 \\ 1\end{array}\right) \cdot\left(\begin{array}{l}1 \\ 1\end{array}\right) \cdot\left(\begin{array}{l}2 \\ 2\end{array}\right)=1$ which is the group de substituents $A_{1} A_{2} A_{3} B_{3}, \quad N_{1,2,3}^{c}=0, \quad N_{1,2,3}^{a+c}=0$.

3) Let us determine the number of substituent groups having 6 substituent types or even 6-tuples of letters, one of which is chosen from $I R_{1}$, one in $I R_{2}$, two in $I R_{3}$ and two in $I R_{4}$.

We have $I R_{1}=\left\{A_{1}\right\}, I R_{2}=\left\{A_{2}\right\}, I R_{3}=\left\{A_{3}, B_{3}\right\}$ and

$$
I R_{4}=\left\{A_{4}, B_{4}, C_{4}, D_{4}, D_{4}^{\prime}\right\} ;
$$

$s_{1}=1, p_{1}=1, s_{1}-p_{1}=0, i_{1}=1, s_{2}=1, p_{2}=1, s_{2}-p_{2}=0, i_{2}=1, s_{3}=2$, $p_{3}=2, s_{3}-p_{3}=0, i_{3}=1, s_{4}=5, p_{4}=3, s_{4}-p_{4}=2, i_{4}=2$

We obtain $N_{1,2,3,4}=\left(\begin{array}{l}1 \\ 1\end{array}\right) \cdot\left(\begin{array}{l}1 \\ 1\end{array}\right) \cdot\left(\begin{array}{l}2 \\ 2\end{array}\right) \cdot\left(\begin{array}{l}5 \\ 2\end{array}\right)=10$ which is the following groups of substituents: $A_{1} A_{2} A_{3} B_{3} A_{4} B_{4}, A_{1} A_{2} A_{3} B_{3} A_{4} C_{4}, A_{1} A_{2} A_{3} B_{3} A_{4} D_{4}, A_{1} A_{2} A_{3} B_{3} A_{4} D_{4}^{\prime}$, $A_{1} A_{2} A_{3} B_{3} B_{4} C_{4}, A_{1} A_{2} A_{3} B_{3} B_{4} D_{4}, A_{1} A_{2} A_{3} B_{3} B_{4} D_{4}^{\prime}, A_{1} A_{2} A_{3} B_{3} C_{4} D_{4}, A_{1} A_{2} A_{3} B_{3} C_{4} D_{4}^{\prime}$, 
$A_{1} A_{2} A_{3} B_{3} D_{4} D_{4}^{\prime} ; \quad N_{1,2,3,4}^{a}=\left(\begin{array}{l}1 \\ 1\end{array}\right) \cdot\left(\begin{array}{l}1 \\ 1\end{array}\right) \cdot\left(\begin{array}{l}2 \\ 2\end{array}\right) \cdot\left(\begin{array}{l}3 \\ 2\end{array}\right)=3$ is the groups of substituents: $A_{1} A_{2} A_{3} B_{3} A_{4} B_{4}, \quad A_{1} A_{2} A_{3} B_{3} A_{4} C_{4}, \quad A_{1} A_{2} A_{3} B_{3} B_{4} C_{4} ; \quad N_{1,2,3,4}^{c}=0 ; \quad N_{1,2,3,4}^{a+c}=10-3=7$ which corresponds to the following groups of substituents: $A_{1} A_{2} A_{3} B_{3} A_{4} D_{4}$, $A_{1} A_{2} A_{3} B_{3} A_{4} D_{4}^{\prime}, A_{1} A_{2} A_{3} B_{3} B_{4} D_{4}, A_{1} A_{2} A_{3} B_{3} B_{4} D_{4}^{\prime}, A_{1} A_{2} A_{3} B_{3} C_{4} D_{4}, A_{1} A_{2} A_{3} B_{3} C_{4} D_{4}^{\prime}$, $A_{1} A_{2} A_{3} B_{3} D_{4} D_{4}^{\prime}$.

\subsection{Calculation of the Number of Chiral and Achiral Skeletons of}

$$
\text { the System } C_{n} H_{m_{0}}\left(\boldsymbol{R}_{k_{1}}\right)_{m_{1}} \cdots\left(\boldsymbol{R}_{k_{\alpha}}\right)_{m_{\alpha}} \cdots\left(\boldsymbol{R}_{k_{\zeta}}\right)_{m_{\zeta}}
$$

The "stereoisomeric" composition of the system

$$
C_{n} H_{m_{0}}\left(R_{k_{1}}\right)_{m_{1}} \cdots\left(R_{k_{\alpha}}\right)_{m_{\alpha}} \cdots\left(R_{k_{\zeta}}\right)_{m_{\zeta}}
$$

results from 1) the nature of alkyl radicals of distinct k-size (chiral or achiral tree) and 2) the manner of attaching or implanting them to the monocycle (chiral or achiral location on the monocycle).

Unlike the polyalkylation of the monocyclic cycloalkane treated by Nemba et al. [23] where we had combinations of alkyl groups of the same order and same steric configuration that is to say homomorphous trees, we perform in this work combinations of heteromorphous alkyl trees having distinct configurations and same order $k$, chiral (c), achiral (a), or mixed $(\mathrm{a}+\mathrm{c})$ which will be planted on the monocyclic cycloalkane. In summary and at the risk of repeating ourselves, we observe that once again we have a composite stereoisomerism due to the intrinsic chirality of the alkylated trees and the extrinsic chirality induced by their position or placement on the monocyclic cycloalkane.

The composite stereoisomerism resulting from the present exercise of heteropolyalkylation of the monocyclic cycloalkane is summarized in Table 3 below.

The different entities of Table 3 integrating the stereospecificity of the heteropolyalkylated monocyclic cycloalkane skeletons are defined as follows:

$A_{c}^{c}\left(n ; m_{0}, m_{1}, \cdots, m_{\alpha}, \cdots, m_{\zeta} ; k_{1}, \cdots, k_{\alpha}, \cdots, k_{\zeta}\right):$ is the number of chiral stereoisomers composed from chiral alkyls (Chiral Radicals-Chiral Placements);

$A_{a}^{c}\left(n ; m_{0}, m_{1}, \cdots, m_{\alpha}, \cdots, m_{\zeta} ; k_{1}, \cdots, k_{\alpha}, \cdots, k_{\zeta}\right):$ is the number of achiral stereoisomers compounded from chiral alkyls (Chiral Radicals-Achiral Placements);

\begin{tabular}{|c|c|c|}
\hline $\begin{array}{l}\text { Chirality or achirality of the } \\
\text { heteropolyalkylated }\end{array}$ & \multicolumn{2}{|c|}{ Combinations of steric configurations of alkyl radicals } \\
\hline $\begin{array}{l}\text { Characteristics of placements } \\
m_{1}, \ldots, m_{\gamma}, \ldots, m_{s k} \text { alkyl trees }\end{array}$ & $\begin{array}{l}\text { All the radicals combined } i \text { to } i \text { are } \\
\text { chiral the radicals combined } i \text { to } i \text { are } \\
\text { achiral }\end{array}$ & $\begin{array}{l}\text { All the radicals combined i to } i \text { are } \\
\text { achiral and chiral }\end{array}$ \\
\hline Chiral placements & $\begin{array}{l}\text { Chiral radicals-Chiral placement } \text { Achiral radicals-Chiral placement } \\
\qquad\left(A_{c}^{c}\right) \\
\left(A_{c}^{a}\right)\end{array}$ & $\begin{array}{l}\text { Achiral and chiral radicals-Chiral } \\
\text { placement }\left(A_{c}^{a+c}\right)\end{array}$ \\
\hline Achiral placements & $\begin{array}{l}\text { Chiral radicals-Achiral placement Achiral radicals-Achiral placement } \\
\qquad\left(A_{a}^{c}\right) \\
\left(A_{a}^{a}\right)\end{array}$ & $\begin{array}{l}\text { Achiral and chiral radicals-Achiral } \\
\text { placement }\left(A_{a}^{a+c}\right)\end{array}$ \\
\hline
\end{tabular}

Table 3. Composite stereoisomerism in heteropolyalkylated monocyclic cycloalkanes [23]. 
$A_{c}^{a}\left(n ; m_{0}, m_{1}, \cdots, m_{\alpha}, \cdots, m_{\zeta} ; k_{1}, \cdots, k_{\alpha}, \cdots, k_{\zeta}\right):$ is the number of chiral stereoisomers composed of achiral alkyl trees (Achiral Radicals-Chiral Placements);

$A_{a}^{a}\left(n ; m_{0}, m_{1}, \cdots, m_{\alpha}, \cdots, m_{\zeta} ; k_{1}, \cdots, k_{\alpha}, \cdots, k_{\zeta}\right):$ is the number of achiral stereoisomers compounded from achiral alkyl trees (Achiral Radicals-Achiral Placements);

$A_{c}^{a-c}\left(n ; m_{0}, m_{1}, \cdots, m_{\alpha}, \cdots, m_{\zeta} ; k_{1}, \cdots, k_{\alpha}, \cdots, k_{\zeta}\right):$ is the number of chiral stereoisomers composed of achiral and chiral alkyl trees (Achiral and chiral radicals-chiral Placements);

$A_{a}^{a-c}\left(n ; m_{0}, m_{1}, \cdots, m_{\alpha}, \cdots, m_{\zeta} ; k_{1}, \cdots, k_{\alpha}, \cdots, k_{\zeta}\right):$ is the number of achiral stereoisomers composed of achiral and chiral alkyl trees (Achiral and chiral radicals-Achiral Placements).

The number of chiral and achiral stereoisomers of a monocyclic cycloalkane heteropolysubstituted by alkyl groups of distinct orders $k$ is directly obtained from the product of the number of groups of substituents $N_{k_{1}, k_{2}, \cdots, k_{\alpha}}^{c}, N_{k_{1}, k_{2}, \cdots, k_{\alpha}}^{a}$, $N_{k_{1}, k_{2}, \cdots, k_{\alpha}}^{a+c}$ by the number of chiral placements $A_{c}\left(n, m_{0}, m_{1}, \cdots, m_{\alpha}, \cdots, m_{\zeta}\right)$ and achiral placements $A_{a}\left(n, m_{0}, m_{1}, \cdots, m_{\alpha}, \cdots, m_{\zeta}\right)$ of alkyl trees on the monocyclic cycloalkane of size $n$.

For this purpose, we recall the results of our recurrence formulas established in previous papers [21] [22] [25] which gives respectively the numbers of chiral and achiral skeletons $A_{c}(n, m)$ and $A_{a}(n, m)$ of the system $C_{n} H_{2 n-m} X_{m}$ and also $A_{c}\left(n, m_{1}, m_{2}, \cdots, m_{i}, \cdots, m_{q}\right)$ and $A_{a}\left(n, m_{1}, m_{2}, \cdots, m_{i}, \cdots, m_{q}\right)$ of the system $C_{n} X_{m_{1}} \cdots Y_{m_{i}} \cdots Z_{m_{q}}$. We can therefore generate relations (5)-(10) which give an inventory of the different varieties of stereoisomers resulting from the heteromorphous polyalkylation of the monocyclic cycloalkane with alkyl trees of distinct order $k$.

$$
\begin{aligned}
& A_{c}^{c}\left(n ; m_{0}, m_{1}, \cdots, m_{\alpha}, \cdots, m_{\zeta} ; k_{1}, \cdots, k_{\alpha}, \cdots, k_{\zeta}\right) \\
&=N_{k_{1}, k_{2}, \cdots, k_{\alpha}}^{c} \times A_{c}\left(n, m_{0}, m_{1}, \cdots, m_{\alpha}, \cdots, m_{\zeta}\right) \\
& A_{a}^{c}\left(n ; m_{0}, m_{1}, \cdots, m_{\alpha}, \cdots, m_{\zeta} ; k_{1}, \cdots, k_{\alpha}, \cdots, k_{\zeta}\right) \\
&=N_{k_{1}, k_{2}, \cdots, k_{\alpha}}^{c} \times A_{a}\left(n, m_{0}, m_{1}, \cdots, m_{\alpha}, \cdots, m_{\zeta}\right) \\
& A_{c}^{a}\left(n ; m_{0}, m_{1}, \cdots, m_{\alpha}, \cdots, m_{\zeta} ; k_{1}, \cdots, k_{\alpha}, \cdots, k_{\zeta}\right) \\
&= N_{k_{1}, k_{2}, \cdots, k_{\alpha}}^{a} \times A_{c}\left(n, m_{0}, m_{1}, \cdots, m_{\alpha}, \cdots, m_{\zeta}\right) \\
& A_{a}^{a}\left(n ; m_{0}, m_{1}, \cdots, m_{\alpha}, \cdots, m_{\zeta} ; k_{1}, \cdots, k_{\alpha}, \cdots, k_{\zeta}\right) \\
&=N_{k_{1}, k_{2}, \cdots, k_{\alpha}}^{a} \times A_{a}\left(n, m_{0}, m_{1}, \cdots, m_{\alpha}, \cdots, m_{\zeta}\right) \\
& A_{c}^{a+c}\left(n ; m_{0}, m_{1}, \cdots, m_{\alpha}, \cdots, m_{\zeta} ; k_{1}, \cdots, k_{\alpha}, \cdots, k_{\zeta}\right) \\
&= N_{k_{1}, k_{2}, \cdots, k_{\alpha}}^{a+c} \times A_{c}\left(n, m_{0}, m_{1}, \cdots, m_{\alpha}, \cdots, m_{\zeta}\right) \\
& A_{a}^{a+c}\left(n ; m_{0}, m_{1}, \cdots, m_{\alpha}, \cdots, m_{\zeta} ; k_{1}, \cdots, k_{\alpha}, \cdots, k_{\zeta}\right) \\
&=N_{k_{1}, k_{2}, \cdots, k_{\alpha}}^{a+c} \times A_{a}\left(n, m_{0}, m_{1}, \cdots, m_{\alpha}, \cdots, m_{\zeta}\right)
\end{aligned}
$$

Therefore, the total number of chiral skeletons of the system 


$$
C_{n} H_{m_{0}}\left(R_{k_{1}}\right)_{m_{1}} \cdots\left(R_{k_{\alpha}}\right)_{m_{\alpha}} \cdots\left(R_{k_{\zeta}}\right)_{m_{\zeta}}
$$

is given by:

$$
\begin{aligned}
& A_{c}^{k_{1}, \cdots, k_{\alpha}, \cdots, k_{\zeta}}\left(n ; m_{0}, m_{1}, \cdots, m_{\alpha}, \cdots, m_{\zeta} ; k_{1}, \cdots, k_{\alpha}, \cdots, k_{\zeta}\right) \\
& =A_{c}^{c}\left(n ; m_{0}, m_{1}, \cdots, m_{\alpha}, \cdots, m_{\zeta} ; k_{1}, \cdots, k_{\alpha}, \cdots, k_{\zeta}\right) \\
& \quad+A_{a}^{c}\left(n ; m_{0}, m_{1}, \cdots, m_{\alpha}, \cdots, m_{\zeta} ; k_{1}, \cdots, k_{\alpha}, \cdots, k_{\zeta}\right) \\
& \quad+A_{c}^{a}\left(n ; m_{0}, m_{1}, \cdots, m_{\alpha}, \cdots, m_{\zeta} ; k_{1}, \cdots, k_{\alpha}, \cdots, k_{\zeta}\right) \\
& \quad+A_{c}^{a+c}\left(n ; m_{0}, m_{1}, \cdots, m_{\alpha}, \cdots, m_{\zeta} ; k_{1}, \cdots, k_{\alpha}, \cdots, k_{\zeta}\right) \\
& \quad+A_{a}^{a+c}\left(n ; m_{0}, m_{1}, \cdots, m_{\alpha}, \cdots, m_{\zeta} ; k_{1}, \cdots, k_{\alpha}, \cdots, k_{\zeta}\right)
\end{aligned}
$$

And the total number of achiral stereoisomers is:

$$
\begin{aligned}
& A_{a}^{k_{1}, \cdots, k_{\alpha}, \cdots, k_{\zeta}}\left(n ; m_{0}, m_{1}, \cdots, m_{\alpha}, \cdots, m_{\zeta} ; k_{1}, \cdots, k_{\alpha}, \cdots, k_{\zeta}\right) \\
& =A_{a}^{a}\left(n ; m_{0}, m_{1}, \cdots, m_{\alpha}, \cdots, m_{\zeta} ; k_{1}, \cdots, k_{\alpha}, \cdots, k_{\zeta}\right)
\end{aligned}
$$

\section{Some Examples of Application, Results and Graphical Representations}

\subsection{Number of Stereoisomers of the Heteropolyalkylated Cyclopropane Having Two Heteromorphous Radicals Methyl $\left(i_{1}=1\right)$ and Ethyl $\left(i_{2}=1\right)$}

We have $I R_{1}=\left\{A_{1}\right\}, I R_{2}=\left\{A_{2}\right\} ; s_{1}=1, p_{1}=1, s_{1}-p_{1}=0, i_{1}=1, s_{2}=1$, $p_{2}=1, \quad s_{2}-p_{2}=0, \quad i_{2}=1 ; \quad N_{1,2}=N_{1,2}^{a}=\left(\begin{array}{l}1 \\ 1\end{array}\right) \cdot\left(\begin{array}{l}1 \\ 1\end{array}\right)=1$ which corresponds to the group of substituents $A_{1} A_{2}, \quad N_{1,2}^{c}=0, \quad N_{1,2}^{a+c}=0$.

The group of substituents $A_{1} A_{2}$ induces heteropolyalkylation of type $C_{3} H_{m_{0}} X_{m_{1}} Y_{m_{2}}$ where $X=A_{1}, Y=A_{2}$ or $X=A_{2}$ and $Y=A_{1}$ and $m_{0}+m_{1}+m_{2}=6$. The numbers of stereoisomers $A_{a}^{a}, A_{c}^{a}, A_{a}^{c}, A_{c}^{c}, A_{a}^{a+c}$ and $A_{c}^{a+c}$ are respectively obtained from the following formulas:

$$
\begin{gathered}
A_{a}^{a}=N_{1,2}^{a} \times A_{a}, \quad A_{c}^{a}=N_{1,2}^{a} \times A_{c}, A_{a}^{c}=N_{1,2}^{c} \times A_{a}, A_{c}^{c}=N_{1,2}^{c} \times A_{c}, \\
A_{a}^{a+c}=N_{1,2}^{a+c} \times A_{a} \text { and } A_{c}^{a+c}=N_{1,2}^{a+c} \times A_{c} .
\end{gathered}
$$

The numbers of stereoisomers corresponding to placements or positions $A_{a}$ and $A_{c}$ of $C_{3} H_{m_{0}} X_{m_{1}} Y_{m_{2}}$ having $m_{0} \neq 0, m_{1} \neq 0, m_{2} \neq 0$ are the same with those of the system $C_{n} X_{m_{1}} Y_{m_{2}} Z_{m_{3}}$ which have been calculated in our previous paper for $n=3$ [21] [22] [25]. They are recalled in the present paper.

The numbers of stereoisomers corresponding to placements or positions $A_{a}$ and $A_{c}$ of $C_{3} H_{m_{0}} X_{m_{1}} Y_{m_{2}}$ having $m_{0}=0, m_{1} \neq 0, m_{2} \neq 0$ are equivalent to those of the system $C_{n} H_{2 n-m} X_{m}$ given in our previous paper for $n=3$ [21] [22] [25].

Using all these data, the inventory of stereoisomers of the heteropolyalkylated cyclopropane having two distinct alkyl trees methyl $\left(i_{1}=1\right)$ and ethyl $\left(i_{2}=1\right)$ is summarized in Table 4. 
Table 4. Number of stereoisomers of the heteropolyalkylated cyclopropane having two distinct alkyl trees methyl $\left(i_{1}=1\right)$ and ethyl $\left(i_{2}=1\right)$.

\begin{tabular}{ccccccccccc}
\hline$m_{0}$ & $m_{1}$ & $m_{2}$ & $A_{a}$ & $A_{c}$ & $A_{a}^{a}$ & $A_{c}^{a}$ & $A_{a}^{c}$ & $A_{c}^{c}$ & $A_{a}^{a+c}$ & $A_{c}^{a+c}$ \\
\hline 1 & 1 & 4 & 1 & 2 & 1 & 2 & 0 & 0 & 0 & 0 \\
1 & 2 & 3 & 2 & 4 & 2 & 4 & 0 & & 0 & 0 \\
2 & 2 & 2 & 4 & 7 & 4 & 7 & 0 & 0 & 0 & 0 \\
0 & 1 & 5 & 1 & 0 & 1 & 0 & 0 & 0 & 0 & 0 \\
0 & 2 & 4 & 2 & 1 & 2 & 1 & 0 & 0 & 0 & 0 \\
0 & 3 & 3 & 2 & 1 & 2 & 1 & 0 & 0 & 0 & 0 \\
\hline
\end{tabular}

Some graphical representations:

$n=3, m_{0}=4, m_{1}=1, m_{2}=1$ :

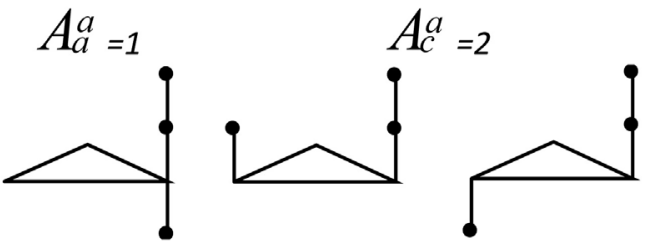

$n=3, m_{0}=3, m_{1}=2, m_{2}=1$ :

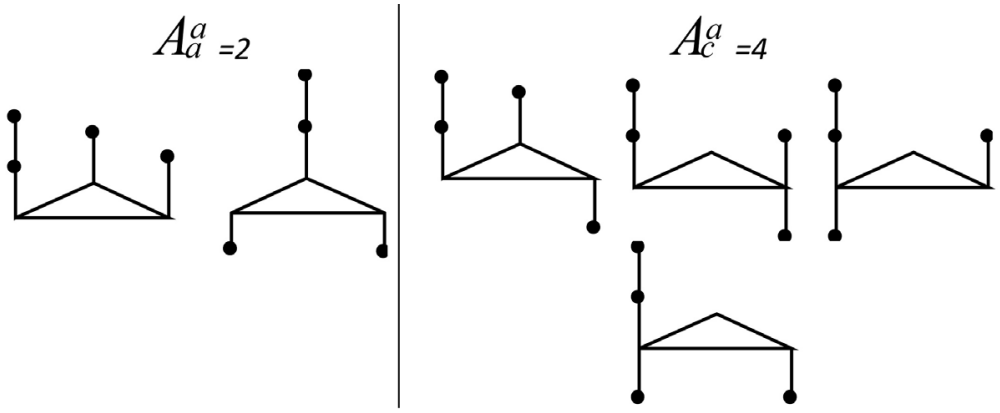

\subsection{Number of Stereoisomers of Heteropolyalkylated}

Cyclobutane Having Two Heteromorphous Radicals Methyl

$\left(i_{1}=1\right)$ and Ethyl $\left(i_{2}=1\right)$

We have $I R_{1}=\left\{A_{1}\right\}, I R_{2}=\left\{A_{2}\right\} ; s_{1}=1, p_{1}=1, s_{1}-p_{1}=0, i_{1}=1, s_{2}=1$, $p_{2}=1, s_{2}-p_{2}=0, i_{2}=1 ; \quad N_{1,2}=N_{1,2}^{a}=\left(\begin{array}{l}1 \\ 1\end{array}\right) \cdot\left(\begin{array}{l}1 \\ 1\end{array}\right)=1$ which corresponds to the group of substituents $A_{1} A_{2}, \quad N_{1,2}^{c}=0, \quad N_{1,2}^{a+c}=0$.

The group of substituents $A_{1} A_{2}$ induces heteropolyalkylation of type $C_{4} H_{m_{0}} X_{m_{1}} Y_{m_{2}}$ with $X=A_{1}, Y=A_{2}$ or $X=A_{2}, Y=A_{1}$ and $m_{0}+m_{1}+m_{2}=8$. The numbers of stereoisomers $A_{a}^{a} A_{c}^{a}, A_{a}^{c}, A_{c}^{c}, A_{a}^{a+c}$ and $A_{c}^{a+c}$ are respectively obtained from the following formulas:

$$
\begin{gathered}
A_{a}^{a}=N_{1,2}^{a} \times A_{a}, A_{c}^{a}=N_{1,2}^{a} \times A_{c}, \quad A_{a}^{c}=N_{1,2}^{c} \times A_{a}, A_{c}^{c}=N_{1,2}^{c} \times A_{c}, \\
A_{a}^{a+c}=N_{1,2}^{a+c} \times A_{a} \text { and } A_{c}^{a+c}=N_{1,2}^{a+c} \times A_{c} .
\end{gathered}
$$


The numbers of stereoisomers corresponding to placements or positions $A_{a}$ and $A_{c}$ of $C_{4} H_{m_{0}} X_{m_{1}} Y_{m_{2}}$ having $m_{0} \neq 0, m_{1} \neq 0, m_{2} \neq 0$ are the same with those of the system $C_{n} X_{m_{1}} Y_{m_{2}} Z_{m_{3}}$ which have been calculated in our previous paper for $n=4$ [21] [22] [25]. They are recalled and used in the present paper.

The numbers of stereoisomers corresponding to placements or positions $A_{a}$ and $A_{c}$ of $C_{4} H_{m_{0}} X_{m_{1}} Y_{m_{2}}$ having $m_{0}=0, m_{1} \neq 0, m_{2} \neq 0$ are equivalent to those of the system $C_{n} H_{2 n-m} X_{m}$ given in our previous paper for $n=4$ [21] [22] [25].

Using all these data, the inventory of stereoisomers of the heteropolyalkylated cyclobutane having two distinct alkyl trees methyl $\left(i_{1}=1\right)$ and ethyl $\left(i_{2}=1\right)$ is summarized in Table 5.

Representation of some graphs.

$n=4, m_{0}=5, m_{1}=2, m_{2}=1$ :
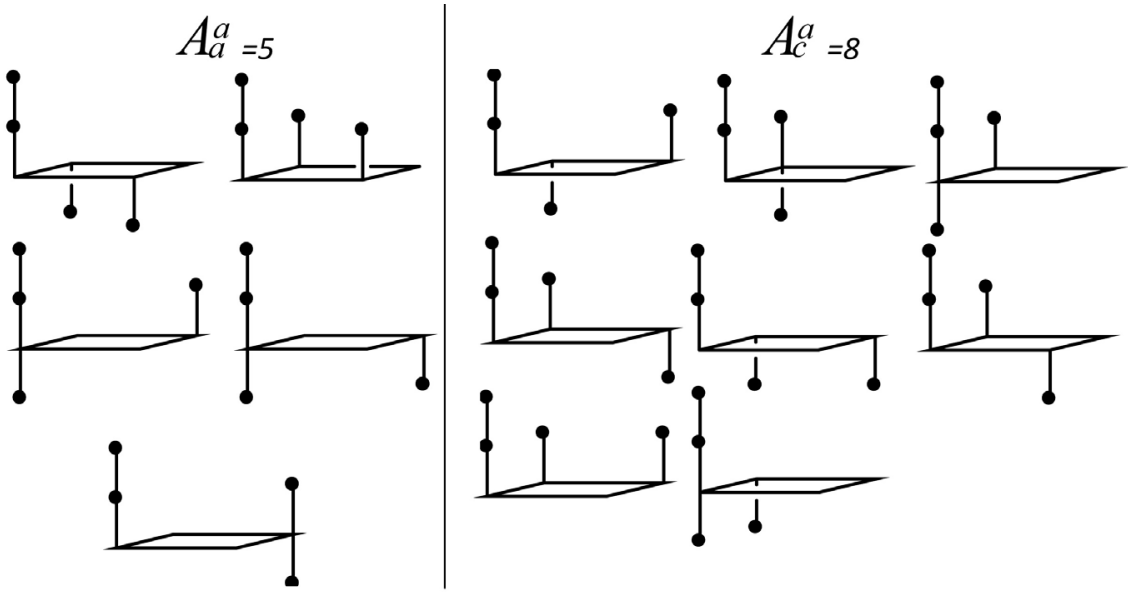

\subsection{Number of Stereoisomers of Heteropolyalkylated Cyclopropane Having Two Heteromorphous Radicals Propyl $\left(i_{3}=1\right)$ and Butyl $\left(i_{4}=1\right)$}

We have $I R_{3}=\left\{A_{3}, B_{3}\right\}, I R_{4}=\left\{A_{4}, B_{4}, C_{4}, D_{4}, D_{4}^{\prime}\right\}, s_{4}=5, p_{4}=3$, $s_{4}-p_{4}=2, i_{4}=1, s_{3}=2, p_{3}=2, s_{3}-p_{3}=0, i_{3}=1$ then $N_{3,4}=\left(\begin{array}{l}2 \\ 1\end{array}\right) \cdot\left(\begin{array}{l}5 \\ 1\end{array}\right)=10$ which corresponds to groups of substituents $A_{3} A_{4}, A_{3} B_{4}$, $A_{3} C_{4}, A_{3} D_{4}, A_{3} D_{4}^{\prime}, B_{3} A_{4}, B_{3} B_{4}, B_{3} C_{4}, B_{3} D_{4}, B_{3} D_{4}^{\prime}, N_{3,4}^{c}=0, N_{3,4}^{a}=\left(\begin{array}{l}2 \\ 1\end{array}\right) \cdot\left(\begin{array}{l}3 \\ 1\end{array}\right)=6$ which corresponds to the groups of substituents $A_{3} A_{4}, A_{3} B_{4}, A_{3} C_{4}, B_{3} A_{4}, B_{3} B_{4}$, $B_{3} C_{4} ; N_{3,4}^{a+c}=10-6=4$ which corresponds to the group of substituents $A_{3} D_{4}$, $A_{3} D_{4}^{\prime}, B_{3} D_{4}, B_{3} D_{4}^{\prime}$.

All groups of substituents give heteropolyalkylations of type $C_{3} H_{m_{0}} X_{m_{1}} Y_{m_{2}}$ such as $m_{0}+m_{1}+m_{2}=6$; with the group de substituents $A_{3} B_{4}$ for example, we can make the assignment $X=A_{3}, \quad Y=B_{4}$ or $X=B_{4}, \quad Y=A_{3}$. Numbers of stereoisomers $A_{a}^{a}, A_{c}^{a}, A_{a}^{c}, A_{c}^{c}, A_{a}^{a+c}$ and $A_{c}^{a+c}$ are respectively obtained from the following formulas: 
Table 5. Number of stereoisomers of heteropolyalkylated cyclobutane having two distinct alkyl trees methyl $\left(i_{1}=1\right)$ and ethyl $\left(i_{2}=1\right)$.

\begin{tabular}{ccccccccccc}
\hline$m_{0}$ & $m_{1}$ & $m_{2}$ & $A_{a}$ & $A_{c}$ & $A_{a}^{a}$ & $A_{c}^{a}$ & $A_{a}^{c}$ & $A_{c}^{c}$ & $A_{a}^{a+c}$ & $A_{c}^{a+c}$ \\
\hline 6 & 1 & 1 & 3 & 2 & 3 & 2 & 0 & 0 & 0 & 0 \\
5 & 2 & 1 & 5 & 8 & 5 & 8 & 0 & & 0 & 0 \\
4 & 2 & 2 & 14 & 23 & 14 & 23 & 0 & 0 & 0 & 0 \\
4 & 3 & 1 & 7 & 14 & 7 & 14 & 0 & 0 & 0 & 0 \\
3 & 3 & 2 & 10 & 30 & 10 & 30 & 0 & 0 & 0 & 0 \\
2 & 3 & 3 & 10 & 30 & 10 & 30 & 0 & 0 & 0 & 0 \\
0 & 7 & 1 & 1 & 0 & 1 & 0 & 0 & 0 & 0 & 0 \\
0 & 6 & 2 & 4 & 1 & 4 & 1 & 0 & 0 & 0 & 0 \\
0 & 5 & 3 & 3 & 2 & 3 & 2 & 0 & 0 & 0 & 0 \\
0 & 4 & 4 & 7 & 3 & 7 & 3 & 0 & 0 & 0 & 0 \\
\hline
\end{tabular}

$$
\begin{gathered}
A_{a}^{a}=N_{4}^{a} \times A_{a}, \quad A_{c}^{a}=N_{4}^{a} \times A_{c}, \quad A_{a}^{c}=N_{4}^{c} \times A_{a}, \quad A_{c}^{c}=N_{4}^{c} \times A_{c}, \quad A_{a}^{a+c}=N_{4}^{a+c} \times A_{a} \\
\quad \text { and } A_{c}^{a+c}=N_{4}^{a+c} \times A_{c} \text { with } N_{3,4}^{a}=6, \quad N_{3,4}^{c}=0 \text { and } N_{3,4}^{a+c}=4 .
\end{gathered}
$$

The numbers of stereoisomers corresponding to placements or positions $A_{a}$ and $A_{c}$ of $C_{3} H_{m_{0}} X_{m_{1}} Y_{m_{2}}$ having $m_{0} \neq 0, m_{1} \neq 0, m_{2} \neq 0$ are the same with those of the system $C_{n} X_{m_{1}} Y_{m_{2}} Z_{m_{3}}$ which have been calculated in our previous paper for $n=3$ [21] [22] [25]. They are recalled in the present paper.

The numbers of stereoisomers corresponding to placements or positions $A_{a}$ and $A_{c}$ of $C_{3} H_{m_{0}} X_{m_{1}} Y_{m_{2}}$ having $m_{0}=0, m_{1} \neq 0, m_{2} \neq 0$ are equivalent to those of the system $C_{n} H_{2 n-m} X_{m}$ given in our previous paper for $n=3$ [21] [22] [25].

Using all these data, the inventory of stereoisomers of the heteropolyalkylated cyclopropane having two heteromorphous radicals propyl $\left(i_{3}=1\right)$ and butyl $\left(i_{4}=1\right)$ is summarized in Table 6.

Some graphical representations:

$n=3, m_{0}=4, m_{1}=1, m_{2}=1$ :

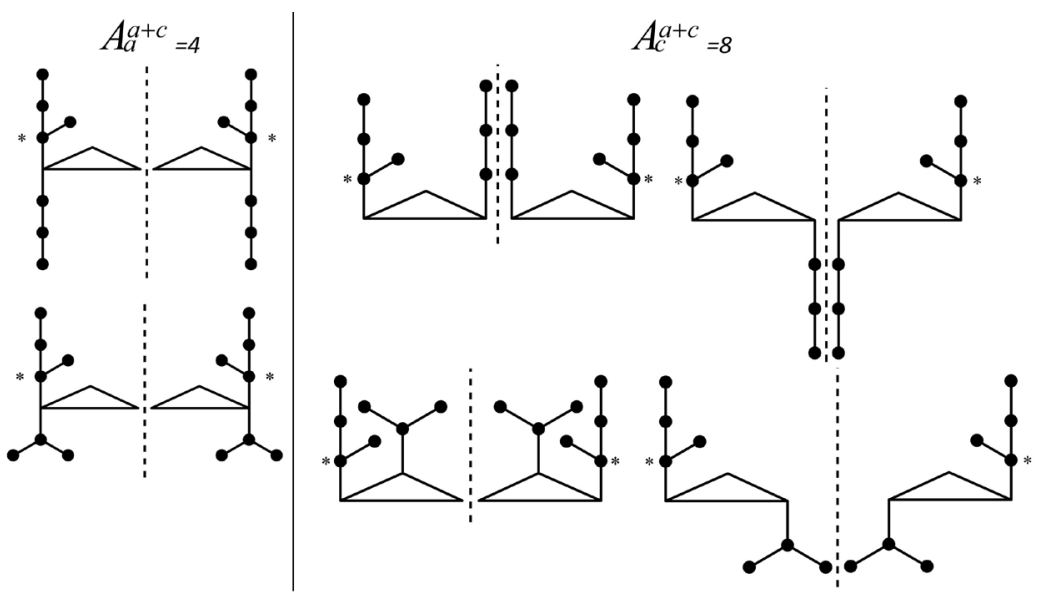


Table 6. Number of stereoisomers of heteropolyalkylated cyclopropane having two heteromorphous radicals propyl $\left(i_{3}=1\right)$ and butyl $\left(i_{4}=1\right)$.

\begin{tabular}{ccccccccccc}
\hline$m_{0}$ & $m_{1}$ & $m_{2}$ & $A_{a}$ & $A_{c}$ & $A_{a}^{a}$ & $A_{c}^{a}$ & $A_{a}^{c}$ & $A_{c}^{c}$ & $A_{a}^{a+c}$ & $A_{c}^{a+c}$ \\
\hline 1 & 1 & 4 & 1 & 2 & 6 & 36 & 0 & 0 & 4 & 8 \\
1 & 2 & 3 & 2 & 4 & 12 & 24 & 0 & 0 & 8 & 16 \\
2 & 2 & 2 & 4 & 7 & 24 & 42 & 0 & 0 & 16 & 28 \\
0 & 1 & 5 & 1 & 0 & 6 & 0 & 0 & 0 & 4 & 0 \\
0 & 2 & 4 & 2 & 1 & 12 & 6 & 0 & 0 & 8 & 4 \\
0 & 3 & 3 & 2 & 1 & 12 & 6 & 0 & 0 & 8 & 4 \\
\hline
\end{tabular}

\subsection{Number of Stereoisomers of Heteropolyalkylated}

\section{Cyclobutane Having Two Heteromorphous Radicals Propyl}

$\left(i_{3}=1\right)$ and Butyl $\left(i_{4}=1\right)$

We have $I R_{3}=\left\{A_{3}, B_{3}\right\}, \quad I R_{4}=\left\{A_{4}, B_{4}, C_{4}, D_{4}, D_{4}^{\prime}\right\}, s_{4}=5, \quad p_{4}=3$ ， $s_{4}-p_{4}=2, i_{4}=1, s_{3}=2, p_{3}=2, s_{3}-p_{3}=0, i_{3}=1$ then $N_{3,4}=\left(\begin{array}{l}2 \\ 1\end{array}\right) \cdot\left(\begin{array}{l}5 \\ 1\end{array}\right)=10$ which corresponds to groups of substituents $A_{3} A_{4}, A_{3} B_{4}$, $A_{3} C_{4}, A_{3} D_{4}, A_{3} D_{4}^{\prime}, B_{3} A_{4}, B_{3} B_{4}, B_{3} C_{4}, B_{3} D_{4}, B_{3} D_{4}^{\prime}, N_{3,4}^{c}=0, N_{3,4}^{a}=\left(\begin{array}{l}2 \\ 1\end{array}\right) \cdot\left(\begin{array}{l}3 \\ 1\end{array}\right)=6$ which corresponds to the group of substituents $A_{3} A_{4}, A_{3} B_{4}, A_{3} C_{4}, B_{3} A_{4}, B_{3} B_{4}$, $B_{3} C_{4} \cdot N_{3,4}^{a+c}=10-6=4$ which corresponds to groups of substituents $A_{3} D_{4}$, $A_{3} D_{4}^{\prime}, B_{3} D_{4}, B_{3} D_{4}^{\prime}$.

The groups of substituents induce heteropolyalkylation of type $C_{4} H_{m_{0}} X_{m_{1}} Y_{m_{2}}$ such as $m_{0}+m_{1}+m_{2}=8$, with the group de substituents $A_{3} B_{4}$ for example, we can make the assignment $X=A_{3}, Y=B_{4}$ or $X=B_{4}, Y=A_{3}$.

The numbers of stereoisomers $A_{a}^{a}, A_{c}^{a}, A_{a}^{c}, A_{c}^{c}, A_{a}^{a+c}$ and $A_{c}^{a+c}$ are respectively obtained from the following formulas:

$$
\begin{gathered}
A_{a}^{a}=N_{3,4}^{a} \times A_{a}, \quad A_{c}^{a}=N_{3,4}^{a} \times A_{c}, \quad A_{a}^{c}=N_{3,4}^{c} \times A_{a}, \quad A_{c}^{c}=N_{3,4}^{c} \times A_{c}, \\
A_{a}^{a+c}=N_{3,4}^{a+c} \times A_{a} \text { and } A_{c}^{a+c}=N_{3,4}^{a+c} \times A_{c}, \\
\text { with } N_{3,4}^{a}=6, \quad N_{3,4}^{c}=0 \text { and } N_{3,4}^{a+c}=4 .
\end{gathered}
$$

The numbers of stereoisomers corresponding to placements or positions $A_{a}$ and $A_{c}$ of $C_{4} H_{m_{0}} X_{m_{1}} Y_{m_{2}}$ having $m_{0} \neq 0, m_{1} \neq 0, m_{2} \neq 0$ are the same with those of the system $C_{n} X_{m_{1}} Y_{m_{2}} Z_{m_{3}}$ which have been calculated in our previous paper for $n=4$ [21] [22] [25]. They are recalled and used in the present paper.

The numbers of stereoisomers corresponding to placements or positions $A_{a}$ and $A_{c}$ of $C_{4} H_{m_{0}} X_{m_{1}} Y_{m_{2}}$ having $m_{0}=0, m_{1} \neq 0, m_{2} \neq 0$ are equivalent to those of the system $C_{n} H_{2 n-m} X_{m}$ given in our previous paper for $n=4$ [21] [22] [25].

Using all these data, the inventory of stereoisomers of the heteropolyalkylated cyclobutane having two heteromorphous radicals propyl $\left(i_{3}=1\right)$ and butyl $\left(i_{4}=1\right)$ is summarized in Table 7 . 
Table 7. Number of stereoisomers of heteropolyalkylated cyclobutane having two heteromorphous radicals propyl $\left(i_{3}=1\right)$ and butyl $\left(i_{4}=1\right)$

\begin{tabular}{ccccccccccc}
\hline$m_{0}$ & $m_{1}$ & $m_{2}$ & $A_{a}$ & $A_{c}$ & $A_{a}^{a}$ & $A_{c}^{a}$ & $A_{a}^{c}$ & $A_{c}^{c}$ & $A_{a}^{a+c}$ & $A_{c}^{a+c}$ \\
\hline 6 & 1 & 1 & 3 & 2 & 18 & 12 & 0 & 0 & 12 & 8 \\
5 & 2 & 1 & 5 & 8 & 30 & 48 & 0 & 0 & 20 & 32 \\
4 & 2 & 2 & 14 & 23 & 84 & 138 & 0 & 0 & 56 & 92 \\
4 & 3 & 1 & 7 & 14 & 42 & 84 & 0 & 0 & 28 & 56 \\
3 & 3 & 2 & 10 & 30 & 60 & 180 & 0 & 0 & 40 & 120 \\
0 & 7 & 1 & 1 & 0 & 6 & 0 & 0 & 0 & 4 & 0 \\
0 & 6 & 2 & 4 & 1 & 24 & 6 & 0 & 0 & 16 & 4 \\
0 & 5 & 3 & 3 & 2 & 18 & 12 & 0 & 0 & 12 & 8 \\
0 & 4 & 4 & 7 & 3 & 42 & 18 & 0 & 0 & 28 & 12 \\
\hline
\end{tabular}

Some graphical representations:

$n=4, m_{0}=6, m_{1}=1, m_{2}=1$ :
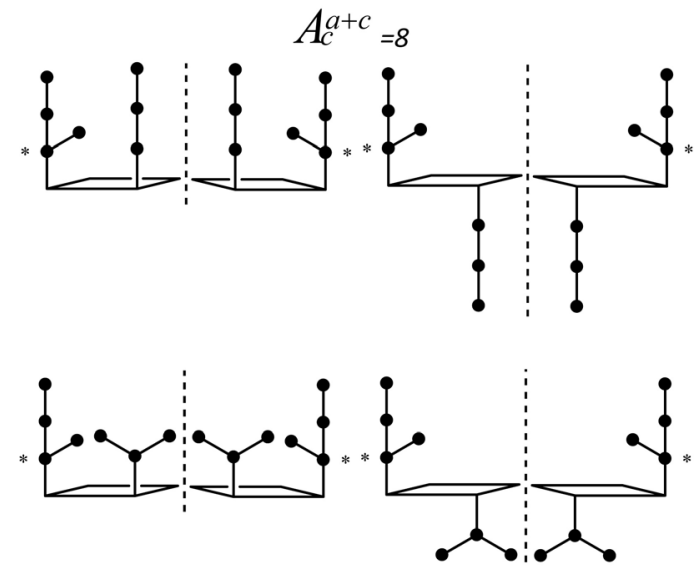

\subsection{Number of Stereoisomers of Heteropolyalkylated Cyclopropane Having Heteromorphous Radicals Propyl $\left(i_{3}=2\right)$ and Butyl $\left(i_{4}=1\right)$}

We have $I R_{3}=\left\{A_{3}, B_{3}\right\}, I R_{4}=\left\{A_{4}, B_{4}, C_{4}, D_{4}, D_{4}^{\prime}\right\}, s_{4}=5, \quad p_{4}=3$, $s_{4}-p_{4}=2, i_{4}=1, s_{3}=2, p_{3}=2, s_{3}-p_{3}=0, i_{3}=2$ then $N_{3,4}=\left(\begin{array}{l}2 \\ 2\end{array}\right) \cdot\left(\begin{array}{l}5 \\ 1\end{array}\right)=5$ which corresponds to groups of substituents $A_{3} B_{3} A_{4}, A_{3} B_{3} B_{4}$, $A_{3} B_{3} C_{4}, A_{3} B_{3} D_{4}, A_{3} B_{3} D_{4}^{\prime} ; \quad N_{3,4}^{c}=0, N_{3,4}^{a}=\left(\begin{array}{l}2 \\ 2\end{array}\right) \cdot\left(\begin{array}{l}3 \\ 1\end{array}\right)=3$ which corresponds to groups of substituents $A_{3} B_{3} A_{4}, A_{3} B_{3} B_{4}, A_{3} B_{3} C_{4} ; N_{3,4}^{a+c}=5-3=2$ which corresponds to groups of substituents $A_{3} B_{3} D_{4}, A_{3} B_{3} D_{4}^{\prime}$.

All those groups of substituents generate heteropolyalkylations of type $C_{3} H_{m_{0}} X_{m_{1}} Y_{m_{2}} Z_{m_{3}}$ such as $m_{0}+m_{1}+m_{2}+m_{3}=6$; with the group of substituents $A_{3} B_{3} C_{4}$ for example, we can make the assignment $X=A_{3}, \quad Y=B_{3}, Z=C_{4}$; 
$X=A_{3}, \quad Y=C_{4}, \quad Z=B_{3} ; \quad X=B_{3}, \quad Y=A_{3}, \quad Z=C_{4} ; \quad X=B_{3}, \quad Y=C_{4}$, $Z=A_{3} ; \quad X=C_{4}, \quad Y=A_{3}, \quad Z=B_{3} ; \quad X=C_{4}, \quad Y=B_{3}, \quad Z=A_{3}$. The numbers of stereoisomers $A_{a}^{a}, A_{c}^{a}, A_{a}^{c}, A_{c}^{c}, A_{a}^{a+c}$ and $A_{c}^{a+c}$ are respectively obtained from the following formulas:

$$
\begin{gathered}
A_{a}^{a}=N_{3,4}^{a} \times A_{a}, A_{c}^{a}=N_{3,4}^{a} \times A_{c}, \quad A_{a}^{c}=N_{3,4}^{c} \times A_{a}, \quad A_{c}^{c}=N_{3,4}^{c} \times A_{c}, \\
A_{a}^{a+c}=N_{3,4}^{a+c} \times A_{a} \text { and } A_{c}^{a+c}=N_{3,4}^{a+c} \times A_{c}, \\
\text { with } N_{3,4}^{a}=3, \quad N_{3,4}^{c}=0 \text { and } N_{3,4}^{a+c}=2 .
\end{gathered}
$$

The numbers of stereoisomers corresponding to placements or positions $A_{a}$ and $A_{c}$ of $C_{3} H_{m_{0}} X_{m_{1}} Y_{m_{2}} Z_{m_{3}}$ having $m_{0} \neq 0, m_{1} \neq 0, m_{2} \neq 0, m_{3} \neq 0$ are the same with those of the system $C_{n} X_{m_{1}} Y_{m_{2}} Z_{m_{3}} U_{m_{4}}$ which have been calculated in our previous paper for $n=3$ [21] [22] [25]. They are recalled in the present paper.

The numbers of stereoisomers of position $A_{a}$ and $A_{c}$ of $C_{3} H_{m_{0}} X_{m_{1}} Y_{m_{2}} Z_{m_{3}}$ having $m_{0}=0, m_{1} \neq 0, m_{2} \neq 0, m_{3} \neq 0$ are equivalent to those of the system $C_{n} X_{m_{1}} Y_{m_{2}} Z_{m_{3}}$ given in our previous paper for $n=3$ [21] [22] [25].

Using all these data, the inventory of stereoisomers of the heteropolyalkylated cyclopropane having two heteromorphous alkyl trees butyl $\left(i_{4}=1\right)$ and propyl $\left(i_{3}=2\right)$ is tabulated in Table 8.

\section{Representation of some graphs.}

$n=3, m_{0}=0, m_{1}=4, m_{2}=1, m_{3}=1$ :
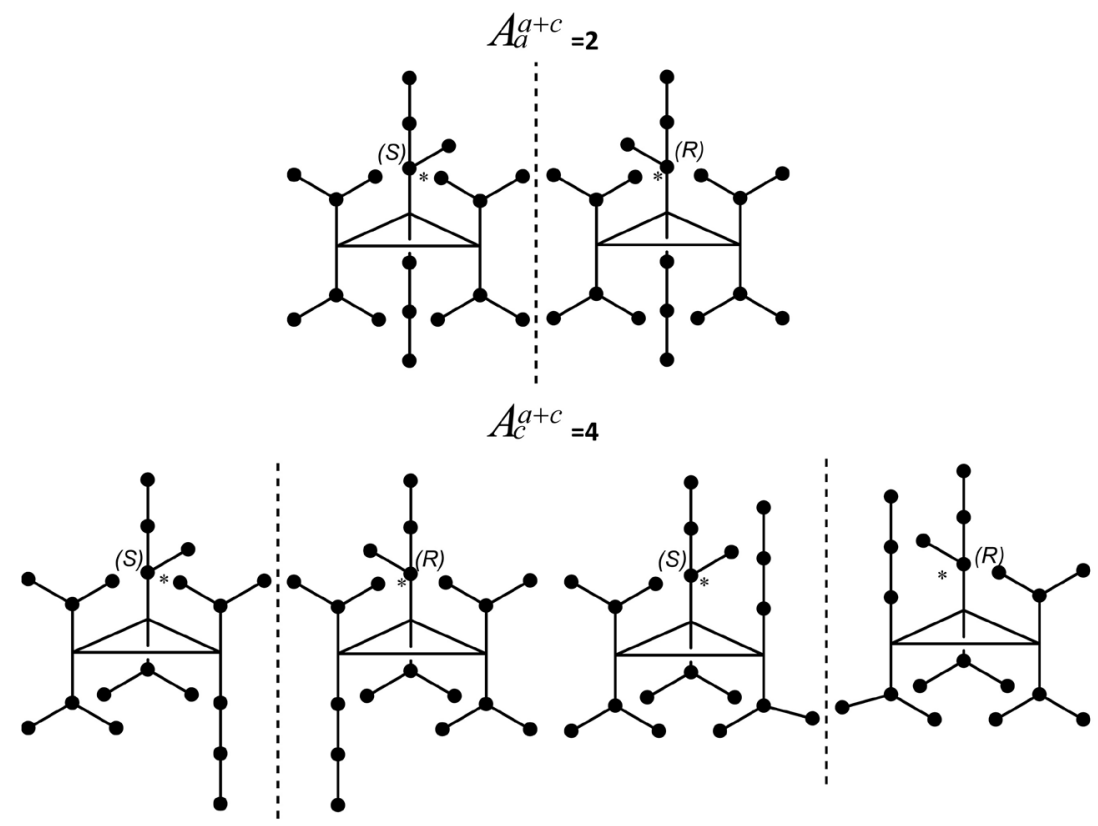

\subsection{Number of Stereoisomers of Heteropolyalkylated Cyclobutane Having Heteromorphous Radicals Propyl $\left(i_{3}=2\right)$ and Butyl $\left(i_{4}=1\right)$ \\ We have $I R_{3}=\left\{A_{3}, B_{3}\right\}, I R_{4}=\left\{A_{4}, B_{4}, C_{4}, D_{4}, D_{4}^{\prime}\right\}, s_{4}=5, p_{4}=3$, $s_{4}-p_{4}=2, i_{4}=1, s_{3}=2, p_{3}=2, s_{3}-p_{3}=0, i_{3}=2$ then}


Table 8. Number of stereoisomers of the heteropolyalkylated cyclopropane having two heteromorphous alkyl trees butyl $\left(i_{4}=1\right)$ and propyl $\left(i_{3}=2\right)$.

\begin{tabular}{cccccccccccc}
\hline$m_{0}$ & $m_{1}$ & $m_{2}$ & $m_{3}$ & $A_{a}$ & $A_{c}$ & $A_{a}^{a}$ & $A_{c}^{a}$ & $A_{a}^{c}$ & $A_{c}^{c}$ & $A_{a}^{a+c}$ & $A_{c}^{a+c}$ \\
\hline 1 & 1 & 1 & 3 & 0 & 10 & 0 & 30 & 0 & 0 & 0 & 20 \\
1 & 1 & 2 & 2 & 2 & 14 & 6 & 42 & 0 & 0 & 4 & 28 \\
0 & 1 & 1 & 4 & 1 & 2 & 3 & 6 & 0 & 0 & 2 & 4 \\
0 & 1 & 2 & 3 & 2 & 4 & 6 & 12 & 0 & 0 & 4 & 8 \\
0 & 2 & 2 & 2 & 4 & 7 & 12 & 21 & 0 & 0 & 8 & 14 \\
\hline
\end{tabular}

$N_{3,4}=\left(\begin{array}{l}2 \\ 2\end{array}\right) \cdot\left(\begin{array}{l}5 \\ 1\end{array}\right)=5$ which corresponds to groups of substituents $A_{3} B_{3} A_{4}, A_{3} B_{3} B_{4}$, $A_{3} B_{3} C_{4}, A_{3} B_{3} D_{4}, A_{3} B_{3} D_{4}^{\prime} ; \quad N_{3,4}^{c}=0, \quad N_{3,4}^{a}=\left(\begin{array}{l}2 \\ 2\end{array}\right) \cdot\left(\begin{array}{l}3 \\ 1\end{array}\right)=3$ which corresponds to the groups of substituents $A_{3} B_{3} A_{4}, A_{3} B_{3} B_{4}, A_{3} B_{3} C_{4} ; N_{3,4}^{a+c}=5-3=2$ which corresponds to groups of substituents $A_{3} B_{3} D_{4}, A_{3} B_{3} D_{4}^{\prime}$.

All those groups of substituents generate heteropolyalkylations of type $C_{4} H_{m_{0}} X_{m_{1}} Y_{m_{2}} Z_{m_{3}}$ such as $m_{0}+m_{1}+m_{2}+m_{3}=8$; with the group de substituents $A_{3} B_{3} C_{4}$ for example, we can make the assignment $X=A_{3}, Y=B_{3}, Z=C_{4}$; $X=A_{3}, \quad Y=C_{4}, \quad Z=B_{3} ; X=B_{3}, \quad Y=A_{3}, \quad Z=C_{4} ; \quad Z=B_{3}, \quad Y=C_{4}$, $Z=A_{3} ; \quad X=C_{4}, \quad Y=A_{3}, \quad Z=B_{3} ; \quad X=C_{4}, \quad Y=B_{3}, \quad Z=A_{3}$.

The numbers of stereoisomers $A_{a}^{a}, A_{c}^{a}, A_{a}^{c}, A_{c}^{c}, A_{a}^{a+c}$ and $A_{c}^{a+c}$ are respectively obtained from the following formulas:

$$
\begin{gathered}
A_{a}^{a}=N_{3,4}^{a} \times A_{a}, \quad A_{c}^{a}=N_{3,4}^{a} \times A_{c}, \quad A_{a}^{c}=N_{3,4}^{c} \times A_{a}, \quad A_{c}^{c}=N_{3,4}^{c} \times A_{c}, \\
A_{a}^{a+c}=N_{3,4}^{a+c} \times A_{a} \text { and } A_{c}^{a+c}=N_{3,4}^{a+c} \times A_{c} \\
\text { where } N_{3,4}^{a}=3, \quad N_{3,4}^{c}=0 \text { and } N_{3,4}^{a+c}=2 .
\end{gathered}
$$

The numbers of stereoisomers corresponding to placements or positions $A_{a}$ and $A_{c}$ of $C_{4} H_{m_{0}} X_{m_{1}} Y_{m_{2}} Z_{m_{3}}$ having $m_{0} \neq 0, m_{1} \neq 0, m_{2} \neq 0, m_{3} \neq 0$ are equivalent to those of the system $C_{n} X_{m_{1}} Y_{m_{2}} Z_{m_{3}} U_{m_{4}}$ which have been calculated in our previous paper for $n=4$ [21] [22] [25]. They are recalled in the present paper.

The numbers of stereoisomers of position $A_{a}$ and $A_{c}$ of $C_{4} H_{m_{0}} X_{m_{1}} Y_{m_{2}} Z_{m_{3}}$ having $m_{0}=0, m_{1} \neq 0, m_{2} \neq 0, m_{3} \neq 0$ are equivalent to those of the system $C_{n} X_{m_{1}} Y_{m_{2}} Z_{m_{3}}$ calculated in our previous paper for $n=4$ [21] [22] [25].

Using all these data, the inventory of stereoisomers of the heteropolyalkylated cyclobutane having heteromorphous radicals propyl $\left(i_{3}=2\right)$ and butyl $\left(i_{4}=1\right)$ is summarized in Table 9.

\subsection{Number of Stereoisomers of Heteropolyalkylated Cyclopropane Having Heteromorphous Radicals Butyl $\left(i_{4}=2\right)$ and Pentyl $\left(i_{5}=2\right)$}

We have $I R_{4}=\left\{A_{4}, B_{4}, C_{4}, D_{4}, D_{4}^{\prime}\right\}, \quad I R_{5}=\left\{A_{5}, B_{5}, C_{5}, D_{5}, E_{5}, E_{5}^{\prime}, F, F_{5}^{\prime}, G, G_{5}^{\prime}\right\}$, $s_{4}=5, \quad p_{4}=3, \quad s_{4}-p_{4}=2, \quad i_{4}=2, \quad s_{5}=11, \quad p_{5}=5, \quad s_{5}-p_{5}=6, \quad i_{5}=2$; 
Table 9. Number of stereoisomers of the heteropolyalkylated cyclobutane having heteromorphous radicals propyl $\left(i_{3}=2\right)$ and butyl $\left(i_{4}=1\right)$.

\begin{tabular}{cccccccccccc}
\hline$m_{0}$ & $m_{1}$ & $m_{2}$ & $m_{4}$ & $A_{a}$ & $A_{c}$ & $A_{a}^{a}$ & $A_{c}^{a}$ & $A_{a}^{c}$ & $A_{c}^{c}$ & $A_{a}^{a+c}$ & $A_{c}^{a+c}$ \\
\hline 1 & 1 & 1 & 5 & 6 & 18 & 18 & 54 & 0 & 0 & 12 & 36 \\
1 & 1 & 2 & 4 & 9 & 48 & 27 & 144 & 0 & 0 & 18 & 56 \\
1 & 1 & 3 & 3 & 12 & 64 & 36 & 192 & 0 & 0 & 24 & 128 \\
1 & 2 & 2 & 3 & 14 & 98 & 42 & 294 & 0 & 0 & 28 & 196 \\
2 & 2 & 2 & 2 & 30 & 150 & 90 & 450 & 0 & 0 & 60 & 300 \\
0 & 6 & 1 & 1 & 3 & 2 & 9 & 6 & 0 & 0 & 6 & 4 \\
0 & 5 & 1 & 2 & 5 & 8 & 15 & 24 & 0 & 0 & 10 & 16 \\
0 & 4 & 2 & 2 & 14 & 23 & 42 & 69 & 0 & 0 & 28 & 46 \\
0 & 4 & 3 & 1 & 7 & 14 & 21 & 42 & 0 & 0 & 14 & 28 \\
0 & 3 & 3 & 2 & 10 & 30 & 30 & 90 & 0 & 0 & 20 & 60 \\
\hline
\end{tabular}

$N_{4,5}=\left(\begin{array}{l}5 \\ 2\end{array}\right) \cdot\left(\begin{array}{l}11 \\ 2\end{array}\right)=550 ; \quad N_{4,5}^{c}=\left(\begin{array}{l}2 \\ 2\end{array}\right) \cdot\left(\begin{array}{l}6 \\ 2\end{array}\right)=15$ which corresponds to groups of substituents $D_{4} D_{4}^{\prime} F_{5} F_{5}^{\prime}, \quad D_{4} D_{4}^{\prime} F_{5} G_{5}, \quad D_{4} D_{4}^{\prime} F_{5} G_{5}^{\prime}, \quad D_{4} D_{4}^{\prime} F_{5} H_{5}, D_{4} D_{4}^{\prime} F_{5} H_{5}^{\prime}$, $D_{4} D_{4}^{\prime} F_{5}^{\prime} G_{5}, \quad D_{4} D_{4}^{\prime} F_{5}^{\prime} G_{5}^{\prime}, \quad D_{4} D_{4}^{\prime} F_{5}^{\prime} H_{5}^{\prime}, \quad D_{4} D_{4}^{\prime} F_{5}^{\prime} H_{5}^{\prime}, \quad D_{4} D_{4}^{\prime} G_{5} G_{5}^{\prime}, \quad D_{4} D_{4}^{\prime} G_{5} H_{5}$, $D_{4} D_{4}^{\prime} G_{5} H_{5}^{\prime}, \quad D_{4} D_{4}^{\prime} G_{5}^{\prime} H_{5}, \quad D_{4} D_{4}^{\prime} G_{5}^{\prime} H_{5}^{\prime}, \quad D_{4} D_{4}^{\prime} G_{5} H_{5}^{\prime} ; \quad N_{4,5}^{a}=\left(\begin{array}{l}3 \\ 2\end{array}\right) \cdot\left(\begin{array}{l}5 \\ 2\end{array}\right)=30 ;$ $N_{4,5}^{a+c}=550-30-15=505$.

All the groups of substituents generate heteropolyalkylations of type $C_{3} H_{m_{0}} X_{m_{1}} Y_{m_{2}} Z_{m_{3}} U_{m_{4}}$ such as $m_{0}+m_{1}+m_{2}+m_{3}+m_{4}=6$; with the group de substituents $A_{4} B_{4} C_{5} D_{5}$ for example, we can make the assignment $X=A_{4}$, $Y=B_{4}, \quad Z=C_{5}, \quad U=D_{5} ; \quad X=A_{4}, \quad Y=C_{5}, \quad Z=D_{5}, \quad U=B_{4} ; \quad X=B_{4}$, $Y=A_{4}, Z=D_{5}, U=C_{5}$ and so on. The numbers of stereoisomers $A_{a}^{a}, A_{c}^{a}$, $A_{a}^{c}, A_{c}^{c}, A_{a}^{a+c}$ and $A_{c}^{a+c}$ are respectively obtained from the following formulas:

$$
\begin{gathered}
A_{a}^{a}=N_{4,5}^{a} \times A_{a}, A_{c}^{a}=N_{4,5}^{a} \times A_{c}, \quad A_{a}^{c}=N_{4,5}^{c} \times A_{a}, \quad A_{c}^{c}=N_{4,5}^{c} \times A_{c}, \\
A_{a}^{a+c}=N_{4,5}^{a+c} \times A_{a} \text { and } A_{c}^{a+c}=N_{4,5}^{a+c} \times A_{c}, \\
\text { with } N_{4,5}^{a}=30, N_{4,5}^{c}=15, N_{4,5}^{a+c}=505 .
\end{gathered}
$$

The numbers of stereoisomers corresponding to placements or positions $A_{a}$ and $A_{c}$ of $C_{3} H_{m_{0}} X_{m_{1}} Y_{m_{2}} Z_{m_{3}} U_{m_{4}}$ having $m_{0} \neq 0, m_{1} \neq 0, m_{2} \neq 0, m_{3} \neq 0$; $m_{4} \neq 0$ are equivalent to those of the system $C_{n} X_{m_{1}} Y_{m_{2}} Z_{m_{3}} U_{m_{4}} V_{m_{5}}$ determined in our previous paper for $n=3$ [21] [22] [25].

The numbers of stereoisomers of position $A_{a}$ and $A_{c}$ of $C_{3} H_{m_{0}} X_{m_{1}} Y_{m_{2}} Z_{m_{3}} U_{m_{4}}$ having $m_{0}=0, m_{1} \neq 0, m_{2} \neq 0, m_{3} \neq 0, m_{4} \neq 0$ are equivalent to those of the system $C_{n} X_{m_{1}} Y_{m_{2}} Z_{m_{3}} U_{m_{4}}$ also derived in our previous paper for $n=3$ [21] [22] [25].

Using all these data, the inventory of stereoisomers of the heteropolyalkylated cyclopropane having heteromorphous alkyl trees butyl $\left(i_{4}=2\right)$ and pentyl $\left(i_{5}=2\right)$ is summarized in Table 10. 
Table 10. Number of stereoisomers of the heteropolyalkylated cyclopropane having heteromorphous alkyl trees butyl $\left(i_{4}=2\right)$ and pentyl $\left(i_{5}=2\right)$.

\begin{tabular}{ccccccccccccc}
\hline$m_{0}$ & $m_{1}$ & $m_{2}$ & $m_{3}$ & $m_{4}$ & $A_{a}$ & $A_{c}$ & $A_{a}^{a}$ & $A_{c}^{a}$ & $A_{a}^{c}$ & $A_{c}^{c}$ & $A_{a}^{a+c}$ & $A_{c}^{a+c}$ \\
\hline 1 & 1 & 1 & 1 & 2 & 0 & 30 & 0 & 900 & 0 & 450 & 0 & 15,150 \\
0 & 1 & 1 & 1 & 3 & 0 & 10 & 0 & 300 & 0 & 150 & 0 & 5050 \\
0 & 1 & 1 & 2 & 2 & 2 & 14 & 60 & 420 & 30 & 210 & 1010 & 7070 \\
\hline
\end{tabular}

\subsection{Number of Stereoisomers of Heteropolyalkylated} Cyclobutane Having Heteromorphous Radicals Butyl $\left(i_{4}=2\right)$ and Pentyl $\left(i_{5}=2\right)$

We have $I R_{4}=\left\{A_{4}, B_{4}, C_{4}, D_{4}, D_{4}^{\prime}\right\}, I R_{5}=\left\{A_{5}, B_{5}, C_{5}, D_{5}, E_{5}, E_{5}^{\prime}, F, F_{5}^{\prime}, G, G_{5}^{\prime}\right\}$, $s_{4}=5, \quad p_{4}=3, s_{4}-p_{4}=2, i_{4}=2, s_{5}=11, \quad p_{5}=5, s_{5}-p_{5}=6, i_{5}=2 ;$ $N_{4,5}=\left(\begin{array}{l}5 \\ 2\end{array}\right) \cdot\left(\begin{array}{c}11 \\ 2\end{array}\right)=550 ; N_{4,5}^{c}=\left(\begin{array}{l}2 \\ 2\end{array}\right) \cdot\left(\begin{array}{l}6 \\ 2\end{array}\right)=15$ which corresponds to groups of substituents $D_{4} D_{4}^{\prime} F_{5} F_{5}^{\prime}, \quad D_{4} D_{4}^{\prime} F_{5} G_{5}, \quad D_{4} D_{4}^{\prime} F_{5} G_{5}^{\prime}, \quad D_{4} D_{4}^{\prime} F_{5} H_{5}, D_{4} D_{4}^{\prime} F_{5} H_{5}^{\prime}$, $D_{4} D_{4}^{\prime} F_{5}^{\prime} G_{5}, \quad D_{4} D_{4}^{\prime} F_{5}^{\prime} G_{5}^{\prime}, \quad D_{4} D_{4}^{\prime} F_{5}^{\prime} H_{5}, \quad D_{4} D_{4}^{\prime} F_{5}^{\prime} H_{5}^{\prime}, \quad D_{4} D_{4}^{\prime} G_{5} G_{5}^{\prime}, \quad D_{4} D_{4}^{\prime} G_{5} H_{5}$, $D_{4} D_{4}^{\prime} G_{5} H_{5}^{\prime}, \quad D_{4} D_{4}^{\prime} G_{5}^{\prime} H_{5}, \quad D_{4} D_{4}^{\prime} G_{5}^{\prime} H_{5}^{\prime}, \quad D_{4} D_{4}^{\prime} H_{5} H_{5}^{\prime} ; \quad N_{4,5}^{a}=\left(\begin{array}{l}3 \\ 2\end{array}\right) \cdot\left(\begin{array}{l}5 \\ 2\end{array}\right)=30$; $N_{4,5}^{a+c}=550-30-15=505$.

All the groups of substituents generate heteropolyalkylations of type $C_{4} H_{m_{0}} X_{m_{1}} Y_{m_{2}} Z_{m_{3}} U_{m_{4}}$ such as $m_{0}+m_{1}+m_{2}+m_{3}+m_{4}=8$; with the group de substituents $A_{4} B_{4} C_{5} D_{5}$ for example, we can make the assignment $X=A_{4}$, $Y=B_{4}, \quad Z=C_{5}, \quad U=D_{5} ; \quad X=A_{4}, \quad Y=C_{5}, \quad Z=D_{5}, \quad U=B_{4} ; \quad X=B_{4}$, $Y=A_{4}, \quad Z=D_{5}, U=C_{5}$ and so on. The numbers of stereoisomers $A_{a}^{a}, A_{c}^{a}$, $A_{a}^{c}, A_{c}^{c}, A_{a}^{a+c}$ and $A_{c}^{a+c}$ are respectively obtained from the following formulas:

$$
\begin{gathered}
A_{a}^{a}=N_{4,5}^{a} \times A_{a}, A_{c}^{a}=N_{4,5}^{a} \times A_{c}, \quad A_{a}^{c}=N_{4,5}^{c} \times A_{a}, \quad A_{c}^{c}=N_{4,5}^{c} \times A_{c}, \\
A_{a}^{a+c}=N_{4,5}^{a+c} \times A_{a} \text { and } A_{c}^{a+c}=N_{4,5}^{a+c} \times A_{c}, \\
\text { with } N_{4,5}^{a}=30, N_{4,5}^{c}=15, N_{4,5}^{a+c}=505 .
\end{gathered}
$$

The numbers of stereoisomers corresponding to placements or positions $A_{a}$ and $A_{c}$ of $C_{4} H_{m_{0}} X_{m_{1}} Y_{m_{2}} Z_{m_{3}} U_{m_{4}}$ having $m_{0} \neq 0, m_{1} \neq 0, m_{2} \neq 0, m_{3} \neq 0$ are equivalent to those of the system $C_{n} X_{m_{1}} Y_{m_{2}} Z_{m_{3}} U_{m_{4}} V_{m_{5}}$ which have been calculated in our previous paper for $n=4$ [21] [22] [25]. They are recalled in the present paper.

The numbers of stereoisomers of position $A_{a}$ and $A_{c}$ of $C_{4} H_{m_{0}} X_{m_{1}} Y_{m_{2}} Z_{m_{3}} U_{m_{4}}$ having $m_{0}=0, m_{1} \neq 0, m_{2} \neq 0, m_{3} \neq 0$ are equivalent to those of the system $C_{n} X_{m_{1}} Y_{m_{2}} Z_{m_{3}} U_{m_{4}}$ calculated in our previous paper for $n=4$ [21] [22] [25].

Using all these data, the inventory of stereoisomers of the heteropolyalkylated cyclobutane having heteromorphous radicals butyl $\left(i_{4}=2\right)$ and pentyl $\left(i_{5}=2\right)$ is summarized in Table 11.

\section{Conclusion}

The combinatorial method developed in the present paper makes it possible to 
Table 11. Number of stereoisomers of heteropolyalkylated cyclobutane having heteromorphous radicals butyl $\left(i_{4}=2\right)$ and pentyl $\left(i_{5}=2\right)$.

\begin{tabular}{ccccccccccccc}
\hline$m_{0}$ & $m_{1}$ & $m_{2}$ & $m_{3}$ & $m_{4}$ & $A_{a}$ & $A_{c}$ & $A_{a}^{a}$ & $A_{c}^{a}$ & $A_{a}^{c}$ & $A_{c}^{c}$ & $A_{a}^{a+c}$ & $A_{c}^{a+c}$ \\
\hline 1 & 1 & 1 & 1 & 4 & 6 & 102 & 180 & 3060 & 90 & 1530 & 3030 & 51,510 \\
1 & 1 & 1 & 2 & 3 & 12 & 204 & 360 & 6120 & 180 & 3060 & 6060 & 103,020 \\
1 & 1 & 2 & 2 & 2 & 18 & 306 & 540 & 9180 & 270 & 4590 & 9090 & 154,530 \\
0 & 1 & 1 & 1 & 5 & 6 & 18 & 180 & 540 & 90 & 270 & 3030 & 9090 \\
0 & 1 & 1 & 2 & 4 & 9 & 48 & 270 & 1440 & 135 & 720 & 4545 & 24,240 \\
0 & 1 & 1 & 3 & 3 & 12 & 64 & 360 & 1920 & 180 & 960 & 6060 & 32,320 \\
0 & 1 & 2 & 2 & 3 & 14 & 98 & 420 & 2940 & 210 & 1470 & 7070 & 49,490 \\
0 & 2 & 2 & 2 & 2 & 30 & 150 & 900 & 4500 & 450 & 2250 & 15,150 & 75,750 \\
\hline
\end{tabular}

directly enumerate enantiomeric pairs and achiral skeletons of the heteropolyalkylated monocyclic cycloalkane $R_{k_{\alpha}}=C_{k_{\alpha}} H_{2 k_{\alpha}+1}$ of which at least two noted alkyl groups each has a distinct order $k_{\alpha}(1 \leq \alpha \leq \zeta) . m_{\alpha}$ is the number of alkyl radicals $R_{k_{\alpha}}$ in the system $C_{n} H_{m_{0}}\left(R_{k_{1}}\right)_{m_{1}} \cdots\left(R_{k_{\alpha}}\right)_{m_{\alpha}} \cdots\left(R_{k_{\zeta}}\right)_{m_{\zeta}}$ satisfying the relationship $\sum_{\alpha=1}^{\zeta} m_{\alpha}+m_{0}=2 n$. This procedure has already been successfully applied by Nemba and Balaban for the case of homomorphic heteropolyalkylation [23] and its advantage is to bypass the Polya method [3] which first requires deriving the cycle index in accordance with symmetry and permutation group of the molecular system and then transform the cycle index into a generating function of the order $2 n$ before continuing with the composition of the graphs to obtain the results of the system $C_{n} H_{m_{0}}\left(R_{k_{1}}\right)_{m_{1}} \ldots\left(R_{k_{\alpha}}\right)_{m_{\alpha}} \ldots\left(R_{k_{\zeta}}\right)_{m_{\zeta}}$. In this work, we have used the basic concepts of permutation group theory and algebraic combinatorics as tools to highlight the chirality and adequacy of achirality induced by composition of molecular systems through heteromorphic polyalkylation. The number of stereoisomers of this family of compounds evolves exponentially as a function of the number of asymmetric carbons of the molecular system carried not only by the radicals but also by the monocyclic cycloalkane. It is worth remembering that the compounds of this family of hydrocarbons serve as starting materials for extensive kinetic and mechanistic studies, while others are used successfully for important applications such as the manufacture of hydrocarbon resins and other chemicals. Hence the interest of the present scientific contribution.

\section{Acknowledgements}

This research was supported by the Ministry of Higher Education of the Republic of Cameroon through the modernization research allowances. Authors also thank the University of Yaounde' I and High Teacher Training College (Cameroon) for the use of their infrastructural facilities. 


\section{Conflicts of Interest}

The authors declare no conflicts of interest regarding the publication of this paper.

\section{References}

[1] Hansen, P.J. and Jurs, P.C. (1988) Chemical Applications of Graph Theory. Isomer Enumeration. Journal of Chemical Education, 65, 662-664. https://doi.org/10.1021/ed065p661

[2] Henze, H.R. and Blair, C.M.J. (1931) Chem. Soc., 53, (a) 3042-3046, (b) 3077-3085.

[3] Pólya, G. (1937) Kombinatorische Abzahlbestimmungen für Gruppen, Graphen und chemische Verbindungen. Acta Mathematica, 68, 145-254.

[4] Burnside, W. (1911) Theory of Groups of Finite Order. 2nd Edition, Cambridge Univ. Press, London.

[5] de Bruijn, N.J. (1964) Polya's Theory of Counting. In: Beckenbach, E.F., Ed., Applied Combinatorial Mathematics, John Wiley, New York, Chapter 5.

[6] Cyvin, S.J., Brunvoll, J.B., Cyvin, N. and Brendsdal, E. (1996) Enumeration of Isomers and Conformers: A Complete Mathematical Solution for Conjugated Polyene Hydrocarbons. Advances in Molecular Structure Research, 2, 213-245. https://doi.org/10.1016/S1087-3295(96)80021-1

[7] Balasubramanian, K. (1979) A Generalized Wreath Product Method for the Enumeration of Stereo and Position Isomers of Poly-Substituted Organic Compounds. Theoretica Chimica Acta, 51, 37-54. https://doi.org/10.1007/BF02399129

[8] Balaban, A.T. (1978) Chemical Graphs. XXXII. Constitutional and Steric Isomers of Substituted Cycloalkanes. Croatica Chemica Acta, 51, 35-42. Balaban, A.T., Kennedy, J.W. and Quintas, L.V. (1988) The Number of Alkanes Having N Carbon and a Longest Chain of Length D, an Application of a Theorem of Polya. Journal of Chemical Education, 65, 304-313. https://doi.org/10.1021/ed065p304

[9] Fowler, P.W. (1995) Isomer Counting Using Point Group Symmetry. Journal of the Chemical Society, Faraday Transactions, 91, 2241-2247. https://doi.org/10.1039/ft9959102241

[10] Hässelbarth, W. and Ruch, E. (1973) Classification of Rearrangement Mechanisms by Means of Double Cosets and Counting Formulas for Numbers of Classes. Theoretica Chimica Acta, 29, 259-268. https://doi.org/10.1007/BF00529052

[11] Harary, F. and Palmer, E. (1973) Graphical Enumeration. Academic Press, New York. https://doi.org/10.1016/B978-0-12-324245-7.50005-8

[12] Kerber, A. (1975) On Graphs and Their Enumeration, Part 1. Math. Chem. Comm., 1, 5-10; (1976) Part II, 2, 17-34. Kerber, A. (1979) Counting Isomers. In: Hinze, J., Ed., The Permutation Group in Physics and Chemistry, Springer-Verlag, Berlin, $1-18$.

[13] Bytautas, L. and Klein, D.J. (1999) Alkane Isomer Combinatorics: Stereostructure Enumeration and Graph Invariant and Molecular Property Distributions. The Journal for Chemical Information and Computer Scientists, 39, 803-808. https://doi.org/10.1021/ci990021g

[14] Lloyd, E.K. (1992) Marks of Permutation Groups and Isomers Enumeration. Journal of Mathematical Chemistry, 11, 207-222. 
https://doi.org/10.1007/BF01164205

[15] Fujita, S. (2000) Systematic Enumeration of Nonrigid Isomers with Given Ligand Symmetries. Journal of Chemical Information and Computer Sciences, 40, 135-146. https://doi.org/10.1021/ci990072d

[16] Nemba, R.M. and Fah, M. (1997) On the Application of Sieve Formula to the Enumeration of the Stable Stereo and Position Isomers of Deoxycyclitols. Journal of Chemical Information and Computer Sciences, 4, 722-725. https://doi.org/10.1021/ci9601291

[17] Nemba, R.M. and Ngouhouo, F. (1994) On the Enumeration of Chiral and Achiral Skeletons of Position Isomers of Homosubstituted Monocyclic Cycloalkanes with a Ring Size N (Odd or Even). Tetrahedron, 50, 6663-6670. https://doi.org/10.1016/S0040-4020(01)89694-0

[18] Nemba, R.M. and Ngouhouo, F. (1994) New Journal of Chemistry, 18, 1175-1182.

[19] Nemba, R.M. and Balaban, A.T. (1998) Algorithm for the Direct Enumeration of Chiral and Achiral Skeletons of Homosubstituted Derivatives of a Monocyclic Cycloalkane with a Large Ring Size N. Journal of Chemical Information and Computer Sciences, 38, 1145-1150. https://doi.org/10.1021/ci980079f

[20] Nemba, R.M. (1996) Solution Générale du problème de dénombrement des stéréoisomères d'un cycloalcane homosubstitué. Comptes rendus de PAcadémie des Sciences, Série II b, 323, 773-779.

[21] Nemba, R.M. and Emadak, A. (2002) Direct Enumeration of Chiral and Achiral Graphs of a Polyheterosubstituted Monocyclic Cycloalkane. Journal of Integer Sequences, 5, Article 02.1.6.

[22] Nemba, R.M. and Emadak, A. (2002) Algorithme de dénombrement des graphes chiraux et achiraux d'un cycloalcane monocyclique hétérosubstitué de formule brute $\mathrm{C} n \mathrm{H} m_{1} \mathrm{Xm}_{2} \mathrm{Ym}_{3}$. Comptes Rendus Chimie, 5, 533-538. https://doi.org/10.1016/S1631-0748(02)01400-5

[23] Nemba, R.M. and Balaban, A.T. (2002) Enumeration of Chiral and Achiral Isomers of an n-Membered Ring with n Homomorphic Alkyl Groups. Match, 46, 235-250.

[24] Emadak, A., Nemba, R.M., Patouossa, I., Ndassa, I.M. and Makon, B.T. (2019) Enumeration of Stereoisomers of Chiral and Achiral Derivatives of Monocyclic Cycloalkanes Having Heteromorphous Alkyl Substituents with the Same Length K. International Journal of Chemical Science, 3, 43-53.

[25] Emadak, A. (2014) Dénombrement des monocycloalcanes hétéropolysubstitués Tome 1: Système ayant une hétéropolysubstitution d'ordre binaire, ternaire, quaternaire, quintuplet et sextuplet. Presse Académique Francophone.

[26] Robinson, R.W., Harary, F. and Balaban, A.T. (1976) The Number of Chiral and Achiral Alkanes and Monosubstituted Alkanes. Tetrahedron, 32, 355-361. https://doi.org/10.1016/0040-4020(76)80049-X 